\title{
AN INVESTIGATION OF FEAR OF THE DARK IN INDIVIDUALS WITH INSOMNIA DISORDER
}

\author{
by \\ Kristin H. G. Maich \\ B.A., McGill University (2008) \\ A thesis \\ presented to Ryerson University \\ in partial fulfillment of the \\ requirements for the degree of \\ Master of Arts \\ in the Program of \\ Psychology
}

B.A.(Hons.), The University of Western Ontario (2013)

Toronto, Canada, 2015

CKristin Maich 2015 


\section{AUTHOR’S DECLARATION FOR ELECTRONIC SUBMISSION OF A THESIS}

I hereby declare that I am the sole author of this thesis. This is a true copy of the thesis, including any required final revisions, as accepted by my examiners.

I authorize Ryerson University to lend this thesis or dissertation to other institutions or individuals for the purpose of scholarly research.

I further authorize Ryerson University to reproduce this thesis or dissertation by photocopying or by other means, in total or in part, at the request of other institutions or individuals for the purpose of scholarly research.

I understand that my thesis may be made electronically available to the public. 


\begin{abstract}
Master of Arts, 2015

Kristin Maich

Psychology

Ryerson University
\end{abstract}

An Investigation of Fear of the Dark in Individuals with Insomnia Disorder

Past research found that individuals with poor sleep report and physiologically exhibit greater fear of the dark (FOD) than good sleepers (Carney et al., 2013). The presence of FOD in individuals with insomnia disorder (ID) would have important implications to our understanding of ID and its treatment. Thus, the present study compared individuals with ID to good sleepers $(N=50)$ on objective and subjective FOD, and explored the FOD construct through participant interviews. Contrary to expectations, ID and good sleeper groups did not differ on FOD measures. Cognitive arousal and trauma history were related to reported dark discomfort. For those with ID, experiences in the dark were characterized by greater sleep effort and pre-sleep arousal generally, but not dark-specific fear per se. For those who were fearful of the dark we posit that there may be a trauma-activated pathway to arousal. Implications and avenues for future research are discussed. 


\section{Acknowledgments}

I would first like to thank my graduate supervisor, Dr. Colleen Carney, for her endless support, guidance, and patience throughout my Master's work. She has invested countless hours into this project, and the expert advice that she has provided over many conversations, always imbued with her sense of humour, has been inspiring.

Thank you to my committee members, Drs. Kelly McShane and Kristin Vickers, for generously contributing their time and thoughtfully reviewing this manuscript. The many conversations I have had with Dr. McShane over the past year as this thesis grew has sparked my curiosity for qualitative research, and I am very grateful for her instrumental feedback on this project.

This project would not have been possible without my co-conspirator and lab mate Angela Lachowski. Thank you also to my lab manager Olya Bogouslavsky and lab mates Dora Zalai and Annabelle Torsein for their support over the past two years. I am lucky to be a part of a lab with such wonderful people!

Finally, I would like to thank my family and friends for their encouragement over the past few years. To my parents, you are my biggest cheerleaders, and I am so grateful for your support. Thank you! 


\section{Table of Contents}

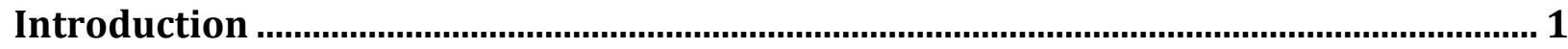

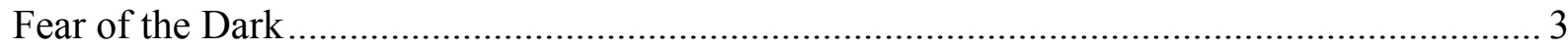

Arousal, Startle, and the Dark ................................................................................. 4

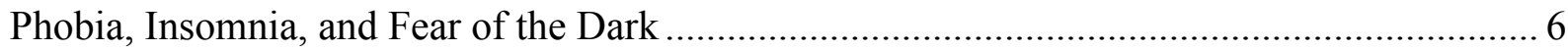

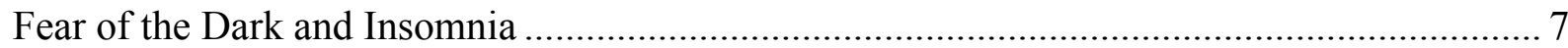

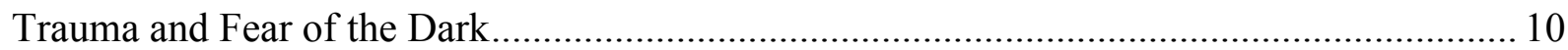

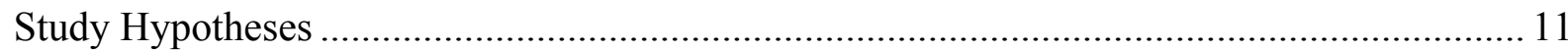

Methods............................................................................................................................... 13

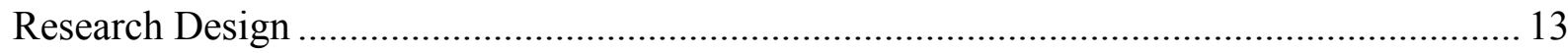

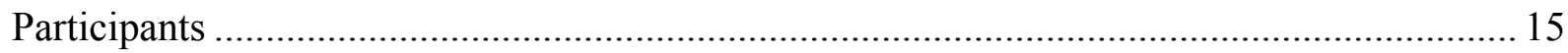

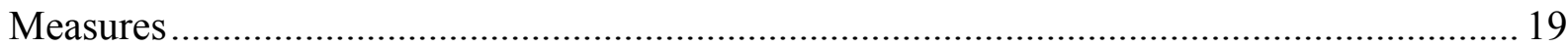

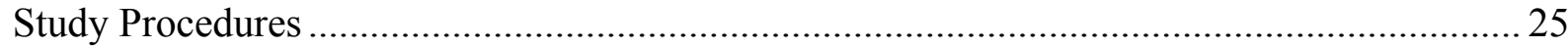

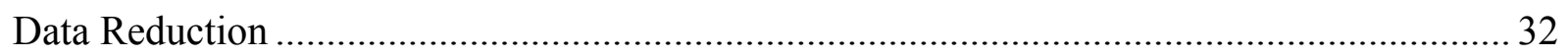

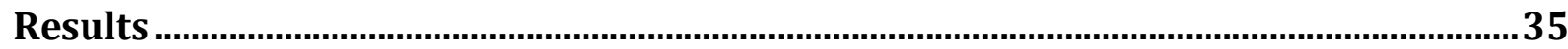

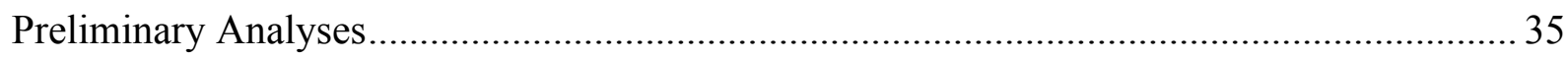

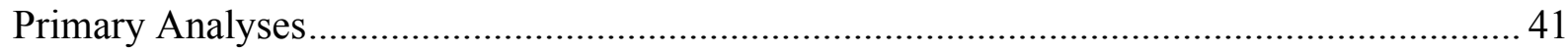

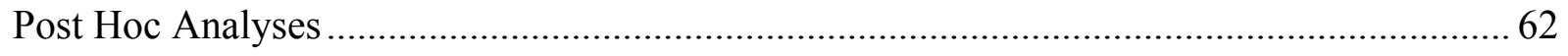

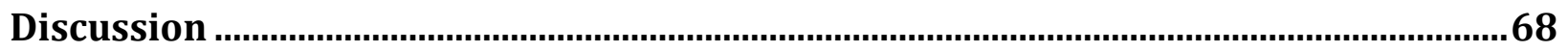

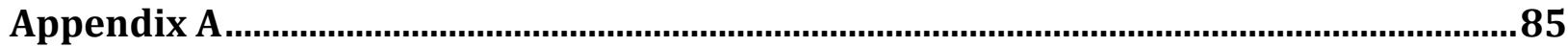

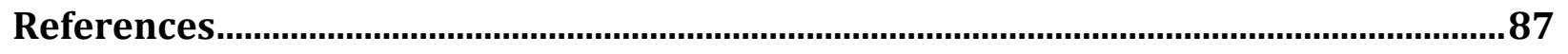




\section{List of Tables}

Table 1: Demographic characteristics of study participants............................ 18

Table 2: Correlations between variables of interest...................................... 37

Table 3: ANOVA assessing group differences on eyeblink amplitude, with age as a covariate.. 39

Table 4: ANOVA assessing group differences on eyeblink latency, with age as a covariate.....40

Table 5: Hierarchical regression analysis predicting insomnia severity from subjective and objective FOD, depression, anxiety, and stress, pre-sleep arousal, and trauma exposure... 49

Table 6: Emergent content domains from the Post-Trial RID Interview.......................54

Table 7: Hierarchical regression analysis predicting discomfort in the dark from eyeblink latency and amplitude, depression, anxiety, and stress, pre-sleep arousal, and trauma exposure...665 


\section{List of Figures}

Figure 1. The startle paradigm, randomized by group.......................................29

Figure 2. Graphical depiction of the interaction found between phase and block on eyeblink amplitude in the startle experiment. ................................................ 45

Figure 3. Emergent theories from thematic analysis of participant responses on the Post-Trial RID interview: The Sleep Performance Pathway and the Trauma Activation

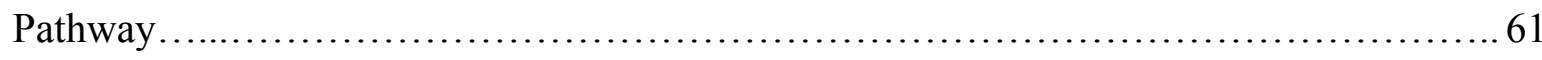




\section{List of Appendices}

Appendix A - Semi-Structured Post-Trial RID Interview.............................85 


\section{Introduction}

Defined as a difficulty with the initiation, maintenance, or self-reported quality of sleep, insomnia is a pervasive disorder that also involves impaired daytime functioning and distress, which are perceived to be detrimental consequences of disrupted sleep (Ohayon, 2002; Pigeon, 2010; Roth \& Ancoli-Israel, 1999). Insomnia is also associated and frequently co-occurs with negative medical (Roth et al., 2011; Rubinstein \& Selwyn, 1998; Stein, Belik, Jacobi, \& Sareen, 2008; Suka, Yoshida, \& Sugimori, 2003) and psychiatric (Mallon, Broman, \& Hetta, 2002;

Ohayon \& Shapiro, 2000; Perlis, Giles, Buysse, Tu, \& Kupfer, 1997; Pigeon et al., 2008)

problems. Evidence from epidemiological research indicates that insomnia affects between 19 and 30 percent of the general adult population, (Leger, Guilleminault, Dreyfus, \& Delahaye, 2000; Morin, LeBlanc, Daley, Gregoire, \& Mérette, 2006), and annual costs (both indirect and direct) associated with insomnia have been found to amount to as much as $\$ 100$ billion in the United States (Fullerton, 2006) and approximately \$6.6 billion in the province of Québec alone (Daley, Morin, LeBlanc, Grégoire, \& Savard, 2009). The Centers for Disease Control and Prevention have labeled sleep problems more generally as a public health epidemic (National Center for Chronic Disease and Prevention and Health Promotion, Division of Adult and Community Health, 2014). Insomnia is thus a potentially debilitating, extremely costly, and highly distressing disorder. While there is currently a great deal of encouraging data related to the treatment of insomnia (e.g., Edinger \& Means, 2005; Edinger, Wohlgemuth, Radtke, Marsh, \& Quillian, 2001; Jacobs, Pace-Schott, Stickgold, \& Otto, 2004; Morgenthaler et al., 2006; Morin, Bootzin, Buysse, Edinger, Espie, \& Lichstein, 2006), there is a critical need for research to address insomnia onset prevention, augment our current understanding of insomnia development, and inform cognitive behavioural treatment options. 
In spite of the high prevalence of and myriad negative consequences associated with insomnia, it is a poorly understood disorder. However, a number of factors are known to contribute to insomnia onset and maintenance. Spielman, Caruso, and Glovinsky (1987) identified a model comprised of three key factors associated with insomnia: predisposing, precipitating, and perpetuating. Biological predisposition or a family history of sleep difficulties are examples of factors that may predispose an individual to the development of insomnia. Precipitating factors may include such things as stressful life events (e.g., divorce, job loss), while perpetuating factors are primarily those beliefs and behaviours that maintain insomnia after onset (e.g., napping, lingering in bed, anxiety about sleep).

Though it is not clear precisely what risk factors are associated with insomnia development, many predisposing factors have been hypothesized in the literature. For example, research suggests that there is a relationship between insomnia and psychological factors such as depression, anxiety, and stress (Taylor, Lichstein, Durrence, Reidel, \& Bush, 2005). Others have hypothesized that a previous experience of trauma may precipitate insomnia onset, which makes sense given that individuals with PTSD tend to have both heightened arousal before bedtime and an increased startle reflex, and many experience sleep-disruptive nightmares (Babson \& Feldner, 2010; Pigeon, Cerulli, Richards, He, Perlis, \& Caine, 2011). Chronic insomnia has also been linked to higher levels of pre-sleep arousal (Harvey, 2002a; LeBlanc, Mérette, Savard, Ivers, Baillargeon, \& Morin 2009; Perlis, Giles, Mendelson, Bootzin, \& Wyatt, 1997). There is support for the importance of conditioned arousal in maintaining insomnia, wherein over time, the bed becomes associated with wakefulness or overall negative affect instead of sleep, and thus triggers insomnia (Freedman \& Sattler, 1982; Perlis et al., 1997). Further, treatments for insomnia typically include a behavioural treatment component of stimulus control that attempts 
to break this association between the bed and arousal. Arousal in this sense is an umbrella term for many constructs, however one possible source or consequence of this arousal may be fear of the dark (FOD). A recent study found that in young adults, FOD is higher among those with sleep problems (Carney, Moss, Atwood, Crowe, \& Andrews, 2013), which raises the question: is FOD characteristic of those with diagnosed insomnia disorder? If FOD is a characteristic that predisposes some individuals to insomnia or perpetuates its chronicity, there are clinical implications for insomnia treatment.

\section{Fear of the Dark}

Fear of the dark is an evolutionarily adaptive mechanism for diurnal creatures (i.e., active during the day, and not at night). Human fear levels are elevated in situations where they are deprived of crucial sensory information (i.e., visual cues). Humans generally exhibit a greater startle response in a dark room compared to a lit room (Grillon, Pellowski, Merikangas, \& Davis, 1997), which suggests that darkness may provoke heightened arousal, or fear. In the absence of light, visual information is decreased, which may decrease a sense of safety or increase feelings of vulnerability and uncertainty. In addition, there is evidence that nocturnal creatures exhibit a greater startle response in the light (Walker, Cassella, Lee, De Lima, \& Davis, 1997), providing further support for the notion that during periods of the day when animals are not typically active, their fear levels are heightened.

FOD is thus not necessarily maladaptive. Moreover, it appears to be neither an unusual nor irrational experience. In children, the dark is one of the most commonly reported fears (Muris, Merckelbach, Mayer, \& Prins, 2000). Recent data collected by the National Sleep Foundation suggest that a proportion of adults may experience discomfort with darkness. The NSF poll data revealed that 11 percent of Americans leave a television on, and 46 percent leave a 
nightlight on in their bedrooms all night (National Sleep Foundation, 2012). Whether lights from the television or in the room are left on to mitigate discomfort in the dark is unknown, but remains one possible explanation.

But what is it about the darkness that induces fearfulness in some individuals? Fear of intruders and/or home invasion is the most commonly reported nighttime fear in children and adolescents (Gordon, King, Gullone, Muris, \& Ollendick, 2007). It is unknown whether this fear of home invasion, or perhaps a more general feeling of a lack of safety, is also the prevailing content of FOD in adults. That is, the content of FOD in adults is currently unknown. It could be discomfort with feeling increased vulnerability or decreased safety, or may perhaps be related to the lack of visual information in the dark, which could increase a sense of uncertainty. FOD may also be related to more specific fears, such as fear of an intruder or home invasion. For those with insomnia, it is possible that fear related to the darkness creates too much anxiety and arousal in bed to allow for the transition into sleep. Given the dearth of research in this area, there is only some literature-derived theory regarding the types of fears adults might experience in the dark or what FOD tends to entail. The present study therefore refined the FOD construct through interview questions and questionnaire items regarding the nature of FOD in adult individuals with insomnia.

\section{Arousal, Startle, and the Dark}

It is well established that many individuals with insomnia experience a phenomenon of heightened physiological and cognitive arousal after turning out the lights in preparation for sleep. The most common type of arousal is conditioned arousal, wherein the individual with insomnia becomes alert in bed after repeated pairings of the sleep environment (e.g., bed) with wakefulness, or arousal, and negative emotion (Perlis et al., 1997). It follows from this model of 
insomnia that individuals with heightened, conditioned pre-sleep arousal experience a conditioned fear not only of the potential for poor sleep involved in the sleep situation, but of other aspects of the sleep situation, such as the darkened room that is typical of bedtime preparation. It is also possible that FOD predisposes an individual to develop insomnia because FOD would create arousal in bed once the lights are turned off.

There is evidence that in humans, fear potentiates the startle reflex (Grillon, Ameli, Woods, Merikangas, \& Davis, 1991; Ross, 1961; Spence \& Runquist, 1958). The notion that the well-established experience of pre-sleep arousal in insomnia may be attributable to a fear of the dark is supported by studies examining mammalian startle reflexes in the dark. An early study of rats found progressive increases in the amplitude of the startle response in the presence of a high level of background noise (Davis, 1974). Davis called this effect sensitization, as the rats were increasingly startled by the acoustic stimuli, rather than habituating to the stimulus presentation over time. Findings indicated that longer exposure periods and high intensity background white noise affected the size of the startle effect, but importantly, when the noise level decreased, the startle effect abated. This early evidence suggests that the environmental condition in which the startle stimulus is presented may affect an organism's experience of startle.

Grillon and colleagues $(1991,1997,1998)$ conducted several investigations of fearpotentiated startle response in humans. One early experimental study found that startle response was greatest across objective measures (e.g., eyeblink amplitude and latency on an electromyogram) when participants were anticipating a negative experience (e.g., electric shock) compared to when anticipation was not present (Grillon et al., 1991). Later, an experimental study utilizing this same startle paradigm found that startle response differed across lighting conditions; participants exhibited a greater startle reflex in the dark than in the light (Grillon et 
al., 1997). A startle paradigm using blinking as a measure of fear is a standard and reliable way

to assess fear because it cannot be "faked"; that is, blinking in response to a stimulus is nearly impossible to inhibit. Cumulatively, the extant research on human response in a startle paradigm suggests that humans experience heightened arousal (i.e., greater startle), in the dark relative to the light, and anticipatory anxiety (i.e., sensitization) increases startle further.

\section{Phobia, Insomnia, and Fear of the Dark}

The expectancy model of fear posits that the motivation to avoid a feared stimulus is a function of both expectation and sensitivity (Reiss, 1991). Cognitions surrounding expectations and sensitivities to the phobic stimulus then trigger avoidance of the feared stimulus. This model is consistent with the maladaptive sleep-related safety behaviours characteristic to insomnia and its maintenance (Harvey, 2002a, 2002b; Hood, Carney, \& Harris, 2011). Though it may be that the avoidant behaviours characteristic to individuals with insomnia are motivated by fear about sleep itself, it may also be that this fear is related to the sleep situation, or sleep environment. That the sleep situation provokes arousal or fear raises the possibility that for some individuals with insomnia, the phobic stimulus is the dark itself.

Based on animal models of fear and anxiety, researchers have suggested that negative emotions are processed by neural structures in the subcortical circuit, which influences reflexes like change in heart-rate and startle (Lang, Davis, \& Ohman, 2000). Though these responses comprise an evolutionarily adaptive defense system for responding to dangerous situations (i.e., triggering an individual to fight against or flee from the dangerous stimulus), specific phobias may have their roots in these responses. Research has found that both the acquisition and expression of conditioned fear involves this defense system (Davis, 1992). When exposed to a previously conditioned fear stimulus, an acoustic startle stimulus prompts a potentiated startle 
response (Davis, 1992; Grillon et al., 1991; Lang, Bradley, Cuthbert, 1990), suggesting that the neural startle circuit is associated with the human fear and defense system (Lang et al., 2000). This defensive system is assumed to be active in the human experience of fear and anxiety, and associated with several of the behaviours consistent with characteristics of individuals with insomnia, such as avoidance, escape, vigilance, and startle (Lang et al., 2000). Though it has been posited that stimuli provoking disgust might trigger a similar startle response, research has shown that the startle reflex is potentiated by feared stimuli, but not disgust stimuli (Balaban \& Taussig, 1994). Thus, it appears that the startle response is somewhat specific and does not include all negative affect.

In line with several of these behaviours, Harvey’s (2002a) cognitive model of insomnia describes five mechanisms that contribute to the maintenance of insomnia: cognitive arousal, or worry; selective attention; misperception of sleep and daytime functioning; unhelpful beliefs about sleep; and sleep-related safety behaviours. The heightened arousal (Grillon et al., 1997) and enhanced startle (Carney et al., 2013) exhibited by some humans in dark conditions is suggestive of fear, and is consistent with the previously described models of phobia proffered by researchers. That is, if the dark is a conditioned fear in some individuals with insomnia, it makes sense according to models of phobia that these individuals engage in defense behaviours such as escape or avoidance (i.e., safety behaviours) and hyperarousal or vigilance, and exhibit a larger startle reflex.

\section{Fear of the Dark and Insomnia}

Fear of the dark is commonly conceptualized as a problem of childhood. However, recent research has suggested that FOD also exists in adults, and may be a significant factor in sleep problems (Carney et al., 2013; Moss, Carney, Atwood, Crowe, \& Luminoso, 2011). A 
study investigating the relationship between poor sleep and discomfort in the dark in a sample of young adults found that poor sleepers both self-reported and physiologically experienced fear of the dark (Carney et al., 2013). On a subjective index, which researchers developed for the purpose of the study, a surprising, statistically significant number of individuals with poor sleep reported FOD. This subjective result was supported by an objective measure of FOD, which assessed the extent to which individuals exhibited physiological reactivity, measured via a startle paradigm, in response to acoustic stimuli presented in a dark room compared to a lit room. Again, the researchers found that the startle effect was larger in the dark condition for poor sleepers than for good sleepers. Moreover, poor sleepers remained more easily startled throughout the study, suggesting at least a lack of habituation, if not a sensitization effect in response to the acoustic stimuli. In contrast, the good sleeper group habituated to the acoustic startle stimuli over time (Carney et al., 2013).

To test whether the effects of the startle response were due to attention, rather than startle, the study incorporated Grillon's (1997) pre-pulse stimuli, paired with the auditory startle stimuli, into the experimental design (Carney et al., 2013). The pre-pulse was a smaller (60 decibel) auditory signal that was presented just before participants heard the larger (102 decibel) startle signal, or pulse, to ensure that the results were not merely due to attentional factors. Analyses indicated that there was no difference in participant response to the pre-pulse stimuli across light and dark conditions. Only the actual pulse stimuli (not the pre-pulse) showed an effect for lighting condition, indicating that the effect found for lighting condition was not due to changes in attention; thus, the pre-pulse did not inhibit the startle response to the pulse.

The finding that individuals with poor sleep experience more fear, in the form of increased startle reflex, in the darkness compared to the light, compared to good sleepers 
suggests that individuals with sleep problems may be experiencing a form of fear or hyperarousal in the sleep situation - that is, exhibiting a conditioned, negative response to the dark. If darkness elicits fearfulness in the form of arousal, an individual who fears the dark may become aversively conditioned to the dark environment that precedes bedtime. The dark may trigger physiological arousal and alertness rather than deactivation, and such individuals may learn to associate the darkened bedroom with wakefulness. This conditioned arousal might then be perpetuated by the cues of the bed and/or switching the light off before bedtime.

In light of evidence from the recent study that demonstrated that FOD is heightened in individuals with poor sleep (Carney et al., 2013), the current study investigated the differential influence of FOD on those with insomnia disorder (ID) compared to good sleepers (controls). In addition to examining FOD in individuals with clinical ID, as opposed to those with reportedly poor sleep, the current study aimed to extend the findings of Carney and colleagues in a more generalizable sample. As detailed below, the current study recruited a much wider age range than the past study by Carney and colleagues and as such, results from the current study were applicable to a broader population. It was expected that individuals with ID would experience higher levels of FOD compared to those with good sleep. To gauge the presence of this construct in participants, fear of the dark was operationalized as reactivity in the dark (RID) in this study, and was assessed through both objective and subjective methods. Though past research found a surprisingly high rate of self-reported fear of the dark in young adults (Carney et al., 2013), it is possible that some adults are wary of the potential for stigma associated with FOD, which is commonly conceptualized as a childhood fear. As such, an objective measure of FOD, as well as a subjective method, was included to convincingly establish the presence of FOD in adults. 


\section{Trauma and Fear of the Dark}

It is known that one symptom of post-traumatic stress disorder (PTSD) is an exaggerated startle response. An experimental startle paradigm study involving Vietnam war veterans found that the magnitude of startle was greater across both dark and light conditions in those diagnosed with PTSD compared with those without PTSD (Grillon, Morgan, Davis, \& Southwick, 1998). Interestingly, results also indicated that the heightened reactivity to the dark condition was not specific to individuals with PTSD, but rather the experience of startle that was facilitated by darkness was common to all the war veteran participants in the study. This heightened sensitivity to darkness, evidenced through heightened startle, was not present in the non-PTSD civilian participants. The researchers suggested that the veterans had become aversively conditioned to the dark environment as a result of their combat experiences. Interestingly, when lighting conditions were held constant, individuals with PTSD continued to experience an exaggerated startle response in this study.

Researchers investigating the relationship between trauma incidence (specifically intimate partner violence) and sleep disturbances found that 46 percent of those with trauma exposure showed clinically significant insomnia (based on a self-report measure of insomnia severity) and 32 percent experienced sleep disturbance in the form of nightmares (Pigeon et al., 2011).

Trauma exposure disrupts normal sleep and is associated with sleep problems (Babson \& Feldner, 2010); moreover, sleep problems predict reduced recovery from PTSD symptoms (e.g., Zayfert \& DeViva, 2004). In light of this evidence and the aforementioned findings, individuals who met criteria for PTSD as assessed during the screening interview were not eligible to participate in the current study. However, given the associations between startle, arousal, and 
trauma exposure, participants in the present study were assessed for past exposure to trauma. It may be that trauma exposure accounts for the relationship between FOD and insomnia severity.

\section{Study Hypotheses}

It was expected that there would be a greater proportion of individuals with FOD in the insomnia group than in the good sleeper group. Further, FOD, as assessed by objective measures, was expected to be significantly greater in individuals with insomnia compared to good sleepers. Compared to good sleepers, those with insomnia were expected to show: a)

decreased eyeblink latency in the dark (measured by EOG), and b) increased eyeblink amplitude in the dark (measured by EOG). Good sleepers and those with insomnia were not expected to differ in the lighted condition.

Habituation is suggested by an increasingly longer period of the time (i.e., greater eyeblink latency) between stimulus presentation and eyeblink over the course of the startle experiment (i.e., between the first and second dark phase). Greater eyeblink latencies in response to stimuli are thus suggestive of habituation. In the light, all participants were expected to habituate over time. Conversely, in conditions eliciting reactivity or fear (i.e., the dark), individuals with high FOD were expected to exhibit sensitization to the white noise stimuli, while the good sleepers were expected to again show habituation. Sensitization is suggested when there is a decreasing period of time between stimuli and eyeblink over the course of the experiment.

Secondly, it was expected that in addition to measures of mood disturbance (i.e., level of depression, anxiety, and stress (DASS-21 score)), pre-sleep arousal (i.e., PSAS-cog and PSASsom scores), and trauma exposure (response to SCID trauma section supplemental questions), FOD (eyeblink latency and eyeblink amplitude) would predict insomnia symptom severity on the 
ISI. Finally, it was expected that in individuals with insomnia certain themes would emerge with respect to FOD in interviews. More specifically, it was expected that individuals with insomnia would report FOD-related thoughts, feelings, and behaviours in the domains of safety, vulnerability, uncertainty, and fear of an intruder or home invasion. 


\section{Methods}

\section{Research Design}

The present study assessed participants' experience of the dark both quantitatively (Part 1), via self-report and a reactivity in the dark (RID) startle paradigm, and qualitatively (Part 2), via a brief Post-Trial RID Interview. Given the large effect size found for differences in measures of objective startle and subjective FOD across sleeper status (good vs. poor) and light condition (dark vs. lit room), power analyses indicated that a minimum sample size of $N=37$ (for the multiple regression analysis) and $N=44$ (for the MANOVA) would be sufficient to obtain adequate power given the planned statistical analyses in the present study. The current study recruited $N=50$ participants.

Part 1 of the study compared individuals with insomnia $(n=25)$ to good sleepers $(n=25)$ on their self-reported and objectively measured fear of the dark (FOD). That is, responses to items on a Dark Discomfort Questionnaire (DDQ) were compared across sleeper status (i.e., insomnia diagnosis vs. good sleeper). Objective measures of reactivity in the dark (i.e., eyeblink latency and amplitude) obtained during a startle paradigm experiment were also compared across sleeper status (between-groups factor) as well as block and phase (within-groups factor) of the experiment. Other variables likely to be related to insomnia (e.g., trauma exposure, pre-sleep arousal, depression, anxiety, stress) were examined in relation to the expected FOD-insomnia association.

Part 2 of the study examined the experience of individuals with insomnia in the dark qualitatively, within a cognitive-behavioural framework. This part of the study investigated the potential presence of certain expected feared stimuli in the dark. Results obtained from this portion of the investigation were considered through a realist lens. 
Realism, in the context of the scientific research, is the broad idea that scientific theories describe the true qualities of phenomena in the world (Maxwell, 2012). Though there are many variants within the realist framework, the prevailing theme is that unlike objectivism, the realist school of thought acknowledges that all knowledge of the world is "partial, incomplete, and fallible" (Maxwell, 2012). That is, for any given phenomenon, there is no single scientifically accurate way of accounting for it. The realist lens thus embraces the idea that there is an 'actual' world, with the caveat that the way in which we as humans understand the world is an inevitably biased construction (Maxwell, 2012). The realist conceptual framework combines hypotheses based on putative explanations with goals around discovering more about what is unknown about a phenomenon. Thus, realism supports research that incorporates the existence of theories about the phenomena being investigated, while also suggesting that other unexpected variables may emerge. The language used to respond to interview questions is considered to be highly related to the experience and meaning of a phenomenon (like FOD) in realism, from which theories can be created (Braun \& Clarke, 2006).

The realist perspective was thus a fitting lens through which to view the phenomenon of fear of the dark. The purpose of the qualitative component (Part 2) of the current study was to refine our current understanding of FOD in the context of insomnia through cognitivebehavioural theory. The perspective taken in the present study was that, based on the relevant literature, there are several hypothesized content areas related to FOD-in particular, safety, intruder or break-in, vulnerability, and uncertainty. However, the present state of knowledge about the way in which these themes may relate to FOD, and how FOD may manifest in those with insomnia, is uncertain, imperfect, and subject to revision. Thus the present study investigated the relationship between FOD, a construct about which little is known, and 
insomnia, while incorporating themes a priori that are expected to emerge in light of past research.

\section{Participants}

The sample consisted of 50 adults between age 18 and $71(M=37.9, S D=15.0)$, including 34 females (68\%) and 16 males (32\%). Additional demographic information for the sample is presented in Table 1.

Participants were recruited from other ongoing research studies involving individuals with insomnia disorder, media advertisements posted around both the Ryerson University campus and the larger community of Toronto, and through referrals from external sleep clinics. Inclusion criteria included both males and females between age 18 and 79 years. Older participants were not included due to age-related changes in humans' sleep architecture. To meet inclusionary criteria for the insomnia group, participants met Research Diagnostic Criteria (RDC-I; Edinger et al., 2004) for insomnia diagnosis (ID). That is, individuals in the ID group reported the existence of insomnia for at least the past month, as per DSM-IV-TR criteria. To better characterize the severity of the insomnia, the Consensus Sleep Diary (CSD) was also used for a one-week baseline monitoring period to derive estimates of mean sleep efficiency (SE); that is, the percent of time asleep while in bed. Only those with SE less than 85 percent were enrolled in the ID group. Additionally, ID group participants obtained a score of greater than the clinical research cutoff of 14 (Buysse, Ancoli-Israel, Edinger, Lichstein, \& Morin, 2006) on the Insomnia Severity Index (ISI). Good sleepers: 1) had no past self-reported history of insomnia; 2) met RDC for good sleeper (Edinger et al., 2004); 3) showed a mean SE of 85 percent or greater on the two-week CSD; and 4) scored less than 14 on the ISI. 
Exclusionary criteria included: the need for immediate psychiatric (e.g., suicidality) or medical care; a recent (past six months prior to screening) suicide attempt; a sleep-disruptive comorbid medical condition; evidence of a comorbid psychological disorder (i.e., borderline personality disorder, bipolar disorder, psychotic or cognitive disorder, or substance abuse) that might unduly influence the participant's sleep, behaviour, or their ability to participate in the study; frequent travel across time zones or work on rotating night shifts; met self-reported criteria for a sleep disorder on the structured diagnostic sleep interview (e.g., circadian rhythm disorder, periodic leg movement disorder, sleep disordered breathing, hypersomnolence); or there was a history of alcohol, narcotic, benzodiazepine, or other substance abuse or dependence in the past six months prior to screening. To reduce possible confounding of participants' response to the startle stimulus in the FOD paradigm, individuals with post-traumatic stress disorder (PTSD) were also excluded, due to the increased reactivity and vigilance typical of those in whom it is diagnosed (Grillon et al., 1998). Individuals could use a variety of prescription or over-the-counter sleep medications throughout the study, allowing results to more accurately mirror the general population of individuals struggling with insomnia. However, if these individuals met criteria for hypnotic dependence (as per the Duke Structured Interview for Sleep Disorders; see Measures section below), they were excluded from the study. If the participant was not hypnotic-dependent, they were asked to remain consistent with their medication over the course of the study (i.e., stop taking the medication or maintain a regular medication dose and schedule).

Identifying participant data was confidential. Participants' signed consent forms were preserved in a locked filing cabinet separate from their individual questionnaire battery and FOD data. All computer files were password protected and identifiable only through participant ID 
numbers. No unique identifiers were included in any of the databases used during analysis of the current study's data. Only those involved in the current study had access to participant data and materials, and all materials were kept within the SAD lab at Ryerson University, which was locked when not in use during the day as well as in the evenings and on weekends. All data will be destroyed 10 years after the publication of the present study. 
Table 1

Demographic characteristics of study participants

\begin{tabular}{|c|c|}
\hline Variable & $n(\%)$ \\
\hline \multicolumn{2}{|l|}{ Sex } \\
\hline Female & $34(68)$ \\
\hline Male & $16(32)$ \\
\hline \multicolumn{2}{|l|}{ Ethnic Background } \\
\hline African Canadian & $2(4)$ \\
\hline East/Southeast Asian & $2(4)$ \\
\hline European Canadian & $33(66)$ \\
\hline South Asian & $3(6)$ \\
\hline Other & $19(38)$ \\
\hline \multicolumn{2}{|l|}{ Living Arrangement } \\
\hline Living alone & $8(16)$ \\
\hline With spouse/partner & $12(24)$ \\
\hline With spouse/partner and children & $9(18)$ \\
\hline With friend(s)/roommate(s) & $6(12)$ \\
\hline With family member(s) & $13(26)$ \\
\hline Other & $1(2)$ \\
\hline \multicolumn{2}{|l|}{ Relationship Status } \\
\hline Single & $24(48)$ \\
\hline Married/common-law & $17(34)$ \\
\hline Live-in partner (less than 2 years) & $3(6)$ \\
\hline Divorced & $3(6)$ \\
\hline Separated & $1(2)$ \\
\hline Widowed & $2(4)$ \\
\hline \multicolumn{2}{|l|}{ Employment Status } \\
\hline Full time & $27(54)$ \\
\hline Part-time & $6(12)$ \\
\hline Not working & $17(34)$ \\
\hline
\end{tabular}




\section{Measures}

Structured Clinical Interview for DSM-IV Axis I and Axis II Disorders

The Structured Clinical Interview for DSM-IV Axis I and Axis II Disorders (SCIDI/SCID-II; First, Spitzer, Gibbon, \& Williams, 2002) are semi-structured, clinician-administered diagnostic interviews used to determine DSM-IV Axis I diagnoses. The SCID has been widely as a diagnostic tool in numerous clinical research trials and clinical psychological assessment, and has good psychometric validity and reliability. The SCID-I was used to exclude individuals who met lifetime criteria for PTSD, bipolar disorder, psychotic disorders or active substance abuse. To better assess for the presence of past criterion A trauma incidence without PTSD diagnosis, the PSTD section of the SCID-I was supplemented with additional questions. These questions were modeled on the PTSD SCID-I question assessing for presence of a criterion A trauma, with the major difference being that this large question was broken down into several questions in the present study and participants were given an opportunity to respond more frequently to potential types of trauma exposure. Further, if a trauma existed, additional questions were posed to ascertain whether the traumatic event took place while sleeping or trying to sleep, or in a bedroom environment. Presence of any trauma was entered into the regression equation as a putative predictor of insomnia severity.

\section{Duke Structured Interview for Sleep Disorders}

The Duke Structured Interview for Sleep Disorders (DSISD; Edinger et al., 2004, 2009) is a tool used to assist with sleep disorder diagnoses. The DSISD has good validity and reliability (Edinger et al., 2009), with kappa values between .71 to .86). This measure was used to differentiate individuals with insomnia from individuals presenting with exclusionary diagnoses including: hypersomnolence, sleep disordered breathing, circadian rhythm disorders, 
periodic limb movement disorder (PLMD), or physiological dependence on prescription hypnotic medication.

\section{Insomnia Severity Index}

The Insomnia Severity Index (ISI; Morin, 1993) is a six-item self-report scale that measures the perceived severity of sleep difficulties such as difficulty falling and staying asleep as well as distress about sleep and impact on daytime functioning. Participants rate each item on a scale from 0 (no sleep difficulty) to 4 (severe sleep difficulty). These ratings are summed to acquire a composite score (between 0 and 28). The recommended cutoff is 14, with scores 15 and above indicating presence of moderate (scores 15-21) or severe (scores 22-28) insomnia (Buysse et al., 2006). There is ample support for the validity and reliability of the ISI in assessing subjective insomnia severity (Bastien, Vallieres, \& Morin, 2001; Morin, Belleville, Belanger, \& Ivers, 2001). In the current study, Cronbach's alpha indicated that the scale items had acceptable reliability $(\alpha=.85)$. ISI score was used as the outcome measure in the regression analysis.

\section{Consensus Sleep Diary}

Sleep diaries are recommended for the subjective assessment of insomnia symptoms over one or two weeks (Buysse et al., 2006). Devised by a group of sleep experts, the Core Consensus Sleep Diary (CSD; Carney et al., 2012) is considered the gold standard measure for subjective assessment of insomnia. It involves the nightly completion of a self-report record form that queries individuals on their bedtime, rise time, and other sleep indices. Data from the CSD yield valuable estimates of an individual's sleep such as sleep onset latency (SOL) and sleep efficiency (SE), and provide detailed information about an individual's sleep, including its severity and variability, schedule, and sleep-wake patterns (Buysse et al., 2006; Carney et al., 
2012). Preliminary psychometric analysis comparing the CSD to an objective measure of sleep (actigraphy) provided support for the validity and diagnostic utility of the CSD in insomnia and healthy populations, and found that unlike actigraph data, it was useful in differentiating normal sleepers from those with clinical insomnia (Maich, Lachowski, Harris, \& Carney, 2013). In the current study, baseline sleep indices including SOL and SE.

\section{Dark Discomfort Questionnaire}

The Dark Discomfort Questionnaire (DDQ; Carney et al., 2013) is a self-report measure designed to assess subjective fear of the dark. The DDQ consists of two subscales. The Discomfort in the Dark Subscale (DDQ-Discomfort) includes 10 items that inquire about individuals' levels of comfort a variety of dark situations (e.g., tunnels, a movie theatre). The Safety Behaviours in the Dark Subscale (DDQ-Behaviours) involves 12 items that inquire about the frequency with which the individual engages in potential sleep-related safety behaviours (e.g., "How many nights per week are you likely to leave a nightlight on in your bedroom?”) and the frequency with which the individual engages in these behaviours in an effort to feel more comfortable. On the DDQ-Discomfort, participants were asked to rate each item pertaining to comfort situations on a Likert-like scale ranging from 0 ("not at all uncomfortable") to 4 (“extremely uncomfortable"). One item in this section specifically queried about the extent to which the individual is uncomfortable in the dark. The item-to-total correlation for this fear of the dark item with the other items on the scale is highly acceptable at $r=.7$ (Carney et al., 2013). The cutoff for current fear of the dark was two or more questions answered as any level of discomfort above 1 ("mildly uncomfortable"). On the DDQ-Behaviours, participants were asked to write the total number of nights per week they engage in the listed safety behaviours. Preliminary psychometric analyses indicate that the DDQ has good internal consistency, with a 
Cronbach's alpha of .90 ( $\alpha=.79$ in the current study). Only the Discomfort subscale was utilized in the current study analyses. In the current study, the DDQ-Discomfort subscale showed acceptable reliability $(\alpha=.87)$. Using both the single item on the DDQ-Discomfort that specifically queries about "the dark" and the total score on this subscale, two chi-square analyses were conducted to examine the proportion of self-reported FOD in individuals with insomnia compared to those with good sleep.

\section{Electrooculography}

Orbicularis Oculi Electrooculography (EOG) activity was recorded via three electrodes placed on participants' faces. Data was collected via an AcqKnowledge software package (Biopac, 2004). Three sterilized disc electrodes were placed around the eye to obtain an accurate idea of eyeblink latency and amplitude after a stimulus presentation. As per in recommended guidelines for measuring human startle via eyeblink (Blumenthal, Cuthbert, Filion, Hackley, Lipp, \& Boxtel, 2005), two leads were attached to small electrodes placed just below the left eye, over the lower orbital portion of the orbicularis oculi muscle, and one ground lead was attached to an electrode placed in the middle of the forehead. The left eye was used because the majority of published startle studies using this system of measurement have used this eye, and research suggests that there are no effects for laterality when stimulation (i.e., sound) is binaural, as in the current study (e.g., Bradley, Cuthbert, \& Lang, 1996, as cited in Blumenthal et al., 2005) RID was assessed via eyeblink latency and amplitude. In similar paradigms (e.g., Grillon et al., 1991, 1997), individuals with low or normal reactivity in a given condition exhibited a smaller magnitude and longer latency of startle response over the course of the experiment compared to those with higher reactivity.

Depression Anxiety Stress Scales 
The Depression Anxiety Stress Scales (DASS-21; Lovibond \& Lovibond, 1995) is a 21item self-report measure to assess its eponymous disorders (i.e., depression, anxiety, and stress). Participants responded to each item (e.g., "I found it difficult to relax") on a 4-point scale ranging from 0 (did not apply to me at all) to 3 (applied to me very much, or most of the time). There is adequate support for the psychometrics of the DASS-21 subscales. The DASS-21 has high internal consistency, with Cronbach's alphas of .94 for depression, .87 for anxiety, and .91 for stress, good concurrent validity, and distinguishes well between depression, anxiety, and stress symptomology (Antony, Bieling, Cox, Enns, \& Swinson, 1998). In the current study, the DASS-21 scale had excellent internal consistency $(\alpha=.96)$. The psychometric properties of the DASS-21 measure have been explored in clinical samples (e.g., Antony et al., 1998; Brown, Chorpita, Kororitsch, \& Barlow, 1997; Clara, Cox, \& Enns, 2001; Page, Hooke, \& Morrison, 2007; Zalai, Sherman, McShane, Shapiro, \& Carney, 2015) and it has been utilized as a measure of depression, anxiety, and stress in samples with insomnia (e.g., Harris, Lack, Wright, Gradisar, \& Brooks, 2007; Moss, Carney, Haynes, \& Harris, 2015; Smith, Kozak, \& Sullivan, 2010). These three mood states were entered in the regression as presumed predictors of insomnia severity.

Pre-sleep Arousal Scale

The Pre-sleep Arousal Scale (PSAS; Nicassio, Mendelowitz, Fussell, \& Petras, 1985) is a 16-item self-report rating scale comprised of two subscales that assess both cognitive (PSAS$\operatorname{cog}$ ) and somatic (PSAS-som) arousal prior to sleep. Participants were asked to indicate the intensity of a range of potential physiological and cognitive symptoms experienced while attempting to fall asleep on a 5-point Likert-type scale, anchored between 0 (not at all) and 5 (extremely). The cognitive subscale includes items such as "depressing or anxious thoughts" and 
"worry about falling asleep." The somatic subscale includes items such as "heart-racing, pounding, or beating irregularity" and "have stomach upset." Scores for each subscale were calculated by summing the response on each item. There is support for the reliability of the PSAS in individuals with insomnia (Nicassio et al., 1985). In the current study, the PSAS had excellent internal consistency $(\alpha=.92)$. Both the somatic and cognitive subscales of the PSAS were entered into the regression equation as potential predictors of insomnia severity. Semi-Structured Post-Trial RID Interview

The Semi-Structured Post-Trial RID Interview was developed for the present study in order to qualitatively examine the nature of participants' experience of the dark and, more specifically, investigate the content of any dark-based fear that emerges. Only individuals from the insomnia group participated in this interview, as it is specifically the FOD experience in individuals with clinical insomnia that the present study investigated.

The Post-Trial RID Interview (see Appendix A) consists of focal questions around FOD. The first question is open-ended ("Typically, when you're in the dark, what is that experience like for you?") and allows for both general and more nuanced responses. This initial question is followed by more specific questions that inquire about the thoughts, emotions, and behaviours surrounding participants' experiences in the dark (e.g., "What kind of thoughts go through your head when you're in the dark?"). Finally, the interview probed for more particular fears that are expected to emerge (e.g., feeling of vulnerability, fear of intruder or break-in) based on fear and phobia literature and studies of FOD in children (e.g., Gordon et al., 2007, Lang et al., 2000). This latter part of the Interview showed acceptable internal consistency in the current study ( $\alpha=$ .83). Verbatim responses obtained were recorded in writing or typing via a laptop by the interviewer, reviewed by participants for accuracy, and subjected to qualitative thematic analysis. 
Punctuation was not recorded as the interviews were obtained in vivo, and as such the interviewer attempted to capture only verbatim content information, rather than inserting information about the intonation, pauses, and emphases of participants' speech.

A thematic analysis of the responses to the Post-Trial RID Interview questions was conducted by the primary investigator using an immersion-crystallization technique. Interview data was obtained from $n=22$ participants in the ID group. A second coder, blinded to study hypotheses and unaffiliated with the Sleep and Depression Lab, was employed to separately code emergent themes in the interviews. Themes that emerged from both the primary investigator and second coder's analyses were then compared and collapsed into content domains. The purpose of this interview was to augment our current understanding of FOD, based on hypotheses regarding this experience in the literature.

\section{Study Procedures}

Participants were part of a larger insomnia treatment study examining the effects of Cognitive Behaviour Therapy for Insomnia (CBT-I) and the potential causal role of cognitive processes in insomnia (CIHR Operating Grant (\#130395): Longitudinal Assessment of Cognitive Reactivity in Insomnia; PI: Carney). Thus participants first completed a brief screening interview by telephone to assess whether preliminary participation criteria were met (e.g., insomnia disorder or good sleeper; between age 18 and 79). Participants attended a screening appointment at the Sleep and Depression (SAD) Laboratory at Ryerson University, where they were assessed for insomnia, other comorbid sleep problems, medical issues, and psychological disorders. Prior to the screening procedures, participants provided written informed consent. Participants then completed the ISI, DASS-21, and a self-report version of the DSISD. This questionnaire battery provided information related to the patient's eligibility, the severity of 
insomnia (if present), and depression, anxiety, and stress levels. The SCID and the DSISD (interview version) were administered to determine participant eligibility, collect information on comorbid psychological disorders, and assess for history of trauma exposure. The duration of the screening procedure was approximately two hours.

During the process of consent for the larger insomnia treatment study, participants were given the opportunity to participate in another study examining physiological reactivity in the dark (RID) and in the light to unexpected noise stimuli. The term "reactivity" was utilized both verbally and in the FOD/RID consent form so as to minimize potential expectancy effects surrounding the notion of "fear of the dark." For example, participants may self-reported differently on the DDQ if they associated fear of the dark with social undesirability or stigma. The physiological label "reactivity" may minimize such expectancy effects. Interested participants received additional information about the RID study and, prior to the experiment proper, completed a second consent form. Participants attended a separate appointment at the SAD lab to complete the study, and did not complete the present study at the time of initial assessment, due to the potential for fatigue. The RID study was completed at some point before participants' CBT-I treatment began so as to ensure that the insomnia treatment did not interfere with individuals' potential startle response or overall arousal during the startle paradigm.

The RID experiment in the present study mirrored the paradigm employed by Carney and colleagues in a similar study with undergraduate student participants (Carney et al., 2013). The entire RID experiment took approximately one hour to complete. Upon arrival at the SAD lab, participants completed consent forms and initial questionnaires prior to being fitted with EOG electrodes and leads, as outlined in the Measures section. Once properly fitted, participants were asked to lie down on the bed in the lab room, place a set of headphones over their ears, and 
attempt keep their eyes open throughout the study (i.e., no dozing or resting with the eyes closed). A trained research assistant or the experimenter began the experiment by starting the audio soundtrack. From a different room, a trained research assistant or the experimenter simultaneously began timing and marked the outset of the experiment within the AcqKnowledge data file. A timer was also started at this time so that the different conditions could be marked in the data file and adjustments could be made to the room's lighting conditions remotely at consistent times. The darkness of the room with the lights off has previously been measured by telephotometer and was consistent with near complete darkness (i.e., $0.06 \mathrm{ft} \mathrm{L}$ ). The headphones canceled out other possible noise interferences.

Once alone in the lab room, participants heard only white background noise from the headphones for the first few minutes. This allowed the experimenter to exit the room without causing undue experiment interference and also allowed the participant to adapt to their initial lighting condition before the acoustic stimuli were presented. The first 6 minutes and 31 seconds of the experiment consisted of the adaptation period, provided so that participants could adapt not only to the lighting but also to the sound of the acoustic stimuli prior to the experimental trial. The acoustic stimuli (bursts of sudden white noise) began approximately three minutes into the adaptation period, and a total of six acoustic stimuli (including 5 pulses and 1 prepulse + pulse) were delivered during this initial adaptation period. The experiment consisted of counterbalanced light and dark conditions. That is, half of the participants (Adaptation Light; AL) were randomly assigned to adapt in a lit room, while half adapted in a dark room (Adaptation Dark; AD). For those in the AD group, the lights were turned off one minute into the adaptation period so as to allow the experimenter to exit the room without interrupting the experiment. The startle paradigm consisted of an adaptation period, which was designed to 
habituate participants to the unexpected startle stimuli, and two blocks of four minutes each, each consisting of two phases (one light, one dark). Group 1 (AD) began with an adaptation period in the dark, followed by light-dark-light-dark phases. Group 2 (AL) began with an adaptation period in the light, followed by dark-light-dark-light phases. The adaptation period was a threeminute presentation of background white noise, followed by six acoustic startle stimuli (bursts of white noise) presented 30 seconds apart (see Figure 1 for a visual representation of the startle paradigm).

The acoustic startle stimuli (the burst of white noise) were presented either alone (S) or $120 \mathrm{~ms}$ after a $30 \mathrm{~ms}$ white noise prepulse (PP). A total of 22 acoustic stimuli (including both pulse and prepulse) were presented across the adaptation period and both blocks. The PP measured participants' prepulse inhibition, and has been used as standard practice in startle paradigms (Carney et al., 2013; Grillon et al., 1997).

Upon completion of the startle paradigm portion of the proposed study, the experimenter or research assistant returned to the experiment room and removed the EOG equipment from the participant. Participants then begin a 10-to-15 minute interview, which consisted of a brief, semi-structured interview assessing FOD (see Appendix B), followed by a questionnaire assessing the nature of participants' experiences during the trial (see Appendix A). Finally, the experimenter or research assistant debriefed participants regarding the nature of the study, explaining that it was an investigation of reactivity in the darkness. After this session, participants received a debriefing letter detailing the purpose of the study, were thanked, and were compensated in the amount of $\$ 10$. 


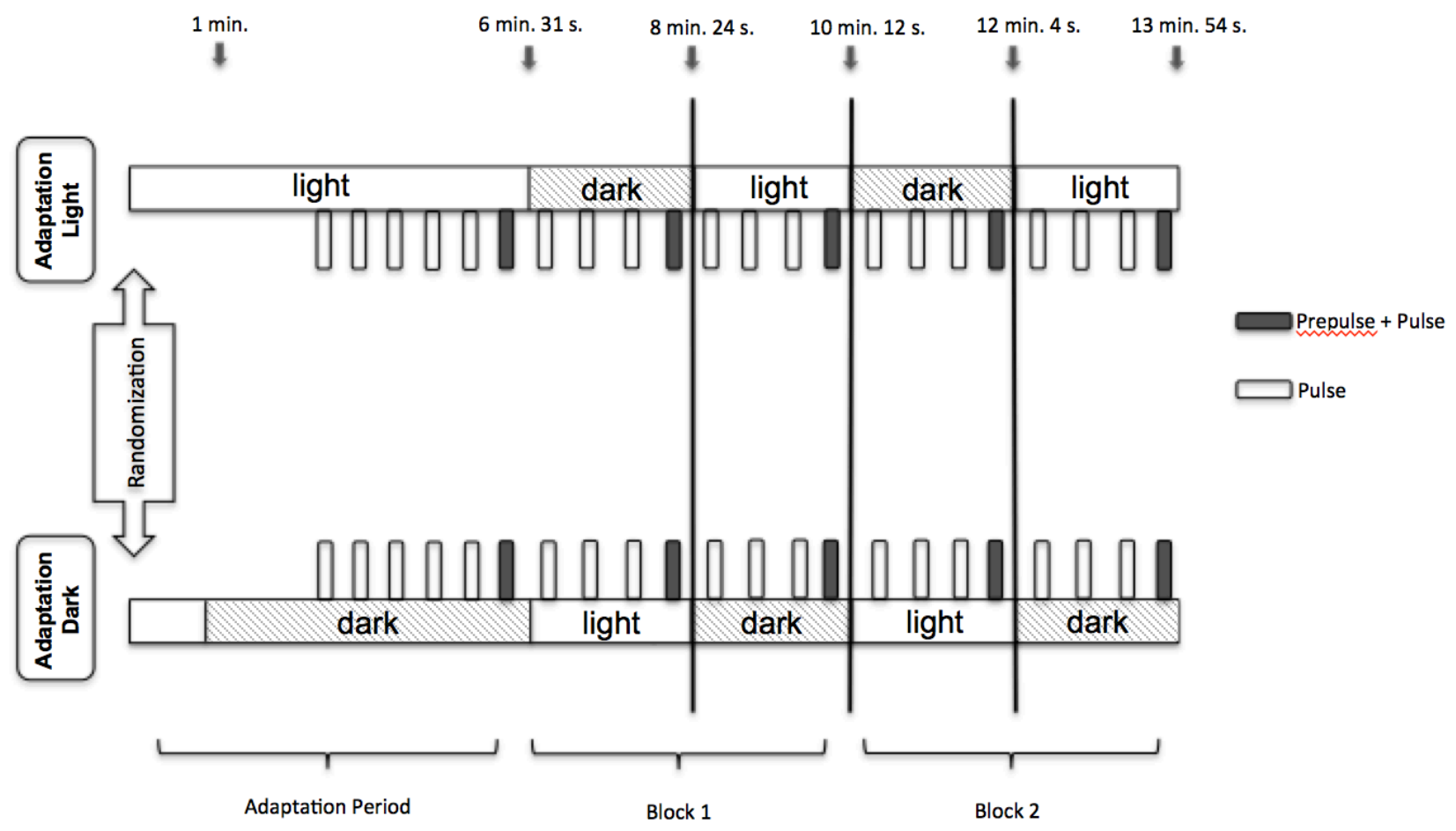

Figure 1. The startle paradigm, randomized by group. 


\section{Data Analyses}

All statistical analyses were conducted using IBM SPSS software (Versions 19.0 and 21.0; SPSS Inc., Chicago, IL, USA).

\section{Preliminary analyses.}

To assess whether the order in which participants received the lighting conditions (i.e., adaptation period occurred in the dark or the light) affected results, a multivariate analysis of variance (MANOVA) was conducted. Nonparametric Spearman's Rho correlational analyses were conducted to compare subjective (DDQ) and objective (eyeblink latency, eyeblink amplitude) FOD. These measures of FOD were also compared to measures of wakefulness on the CSD (i.e., SOL, SE), insomnia severity (ISI score), age, depression, anxiety, and stress (DASS-21), cognitive and somatic pre-sleep arousal (PSAS), and trauma exposure. A chi-square analysis was conducted to assess whether self-reported FOD (i.e., response to the FOD item on the DDQ) was associated with sleeper status (i.e., insomnia vs. good sleeper) significantly more than could be expected by chance alone. To examine whether participant age accounted for any differences found between groups in the primary analyses, age was entered as a covariate in two analyses of variance (ANOVAs) examining group differences on the objective eyeblink indices. A one-way ANOVA with age as a binary variable was conducted to assess for age differences (across groups) on the eyeblink indices.

\section{Primary Analyses.}

Two three-way mixed ANOVAs were then conducted to examine the magnitude of startle (i.e., amplitude of the eyeblink) and reaction time (i.e., eyeblink latency) across sleeper status. The between-groups factor was the participant's group (ID or GS), while the within-groups factors were the time block ( 1 or 2 ) and the phase (i.e., lighting condition; 1 , dark or 2, light). 
To examine the linear relationship between existing causal factors in insomnia and FOD, a multiple hierarchical regression analysis was conducted. The FOD variables (e.g., DDQ score, eyeblink latency, and eyeblink amplitude in second dark phase) were entered into the regression equation first because there is an established literature on the relationship between pre-sleep arousal, trauma, depression, anxiety, and stress in insomnia, thus, it was not necessarily expected that FOD would predict insomnia severity above and beyond the predictive power of these existing factors. FOD was entered in the second step along with the putative factors from step 1. The outcome variable in the regression equation was insomnia severity (ISI score).

\section{Qualitative analysis.}

It was primarily the data from those participants with insomnia disorder who also experienced RID (i.e., objective FOD) that was of interest in the present study, and therefore only data from the ID group were subjected to analysis in Part 2 of this study. Responses to the Semi-Structured Post-Trial RID Interview were examined using thematic analysis. This form of analysis broadly involves the investigation for particular themes in a set of data (Braun \& Clarke, 2006), though unexpected themes may also emerge. Thematic analysis can be used in a variety of different frameworks or lenses. The present study employed a realist framework, and therefore the thematic analysis used the method of highlighting experiences, meanings, and overall "reality" of participants' responses to questions asking about their experiences in the dark. Semantic themes were examined patterns in the data set. That is, the approach was to identify themes that are explicitly stated in participant responses, without researcher interpretation of underlying or latent content in the response data. This analysis involved organizing the data by way of semantic content so that patterns may become clear, followed by interpretation of the importance of the emergent patterns in light of this study's literature-based 
hypotheses. The prevalence of expected and unexpected themes could contribute to the relative importance of that particular theme, but relative proportions were not the only consideration and researcher judgment was also employed to determine the presence of themes, as per Braun and Clarke's recommendations (2006).

An immersion-crystallization technique was used to code the data. Immersioncrystallization is a process that is widely used in qualitative research, particularly when theories or hypotheses about expected themes in the data are present (DiCicco-Bloom \& Crabtree, 2006). Essentially, the process involves a first step of "immersion," wherein the primary investigator becomes immersed in the collected data by reading all of the responses to the interview questions in detail, several times across many days (Borkan, 1999). Once familiarized with this data set, the "crystallization" process began, wherein the primary investigator, for a period of time, suspended this reading process and began to articulate notable themes revealed in the data set. Because the interview was expected to elicit particular response themes around thoughts, feelings, and behaviours, it was expected that patterns would emerge in at least these three broad domains. Outcomes from these analyses were used to interpret the relative importance of various content domains (both hypothesized and unexpected) that emerged from the qualitative interview data.

\section{Data Reduction}

To prepare data from the RID procedure for statistical analysis, EOG activity was first filtered to minimize non-physiological phenomena such as signal drift and environmental noise. A band pass filter was used to filter that data (low pass $0.3 \mathrm{Hertz}(\mathrm{Hz})$, high pass $35.0 \mathrm{~Hz}$ ). The sampling rate was $1000 \mathrm{~Hz}$. A procedure outlined by Grillon and colleagues $(1991,1997)$ was then followed. The location of the white noise startle stimuli on the audio was pinpointed via 
visual scan. Then, for each of the 22 acoustic stimuli on the recording, the latency and amplitude of the eyeblink (EOG) response were extracted from the file while offline using an algorithm developed for the current study using a commercial software package (MATLAB 8.4, The MathWorks Inc., Natick, MA, 2000). This procedure was repeated to access the RID data of each participant.

Both eyeblink latency and peak amplitude were determined in the $20-120 \mathrm{~ms}$ period following the onset of the acoustic startle stimulus. Eyeblink latency was scored as the length of time in milliseconds (ms) between the acoustic stimulus presentation and the point at which the participant's eyeblink, moved outside a pair of amplitude thresholds (measured by the EOG electrodes). These amplitude thresholds were determined by computing the mean of the baseline amplitude, plus or minus two standard deviations of this mean. The baseline amplitude (baseline window) was considered to be the $50 \mathrm{~ms}$ region immediately preceding the presentation of the startle stimulus (Carney et al., 2013, Grillon et al., 1991, 1997). In order to include latencies that fell outside the 20-120 ms period (and therefore did not exhibit a post-stimulus 'startle'), missing latency data was coded "response after $121 \mathrm{~ms}$ " in order to include these long reaction times (i.e., non-startles) in mean latency calculations. In accordance with previous startle response studies, latencies longer than one second (1000 ms) were excluded from analysis (Carney et al, 2013; Grillon et al., 1997), because such latencies are statistically more likely to reflect normal (i.e., non-stimuli related) blinking.

Amplitude (in arbitrary units; $0.0006 \mathrm{mV} /$ arbitrary unit) was computed relative to the average amplitude values recorded in the $50 \mathrm{~ms}$ window of time immediately prior to the onset of the acoustic stimuli. Peak amplitudes were considered to be the local maximum or minimum with the largest absolute amplitude within the target window (i.e., 20-120 ms after stimulus 
onset). The response criterion for a peak was set at 10 arbitrary units (i.e., $0.006 \mathrm{mV}$ ) in accordance with Grillon and colleagues (1991). Amplitudes equal to or greater than $.006 \mathrm{mV}$ were included in the mean amplitude calculations for each phase. Amplitudes that did not meet this cutoff for a 'peak' were recoded as 0 (i.e., no amplitude). 


\section{Results}

\section{Preliminary Analyses}

The distributions of the variables of interest were examined to ascertain whether the assumption of normality was met. Using the Kolmogorov-Smirnov test of normality it was found that there were some variables with a non-normal distribution. Some questionnaire data violated the normality assumption, although this was expected because the questionnaires utilized Likert-like rating scales, producing ordinal data. Nonetheless, analyses of variance (ANOVAs) and multiple regression (Field, 2009) have been found to be robust with non-normal ordinal data (e.g., Sullivan \& D’Agostino, 2003) and non-normal data more generally (see Gardner \& Tremblay, 2007 for recommendations), thus, these data were not transformed.

\section{Order effects.}

A MANOVA was conducted to ensure that the order in which participants received the phases (light first, $n=27$; dark first, $n=23$ ) did not affect reactivity in the dark. Results showed no main effect for order on eyeblink amplitude in either of the first $(F(1,46)=0.76, p=.39)$ or second $(F(1,46)=0.14, p=.71)$ dark phases, nor in the first $(F(1,46)=0.80, p=.38)$ or second $(F(1,46)=1.34, p=.25)$ light phases. The effect sizes $(r=.05-.16)$ were all in the small range according to Cohen's (1992) criteria. There was also no order-by-group (i.e., lighting condition by sleeper status) interaction effect across the dark in light phases in Block 1 or Block $2(p>.25)$. Results from the second ANOVA assessing the effect of order (phase) on eyeblink latency showed no order effect (Dark phase: Block 1, $F(1,46)=0.01, p=.94$; Block 2, $F(1,46)$ $=.03, p=.86 ;$ Light phase: Block $1, F(1,46)=.36, p=.55$, Block $2, F(1,46)=.07, p=.79)$, and there were no order-by-group interactions $(p=.22-.75)$. 
Correlations. Nonparametric Spearman's Rho correlation coefficients were calculated to assess relationships between the variables of interest. Variables examined included the CSD sleep indices (SOL, SE), insomnia severity (ISI score), pre-sleep arousal (PSAS-cog, PSASsom), depression, anxiety, and stress (DASS-21), subjective FOD (DDQ-Discomfort total score), and the frequency of specific thoughts and feelings in the dark (part 2 of the Post-Trial RID Interview), see Table 2. 
Table 2

Spearman's Rho correlations between study variables of interest

\begin{tabular}{|c|c|c|c|c|c|c|c|c|c|c|c|c|c|c|}
\hline Variable & 1 & 2 & 3 & 4 & 5 & 6 & 7 & 8 & 9 & 10 & 11 & 12 & 13 & 14 \\
\hline $\begin{array}{l}\text { 1. Post-RID } \\
\text { Interview }\end{array}$ & 1.00 & & & & & & & & & & & & & \\
\hline $\begin{array}{l}\text { 2. DDQ- } \\
\text { Discomfort }\end{array}$ & $.46^{*}$ & 1.00 & & & & & & & & & & & & \\
\hline 3. ISI & -.02 & .17 & 1.00 & & & & & & & & & & & \\
\hline 4. DASS-D & -.06 & $.31 *$ & $.69 * * *$ & 1.00 & & & & & & & & & & \\
\hline 5. DASS-A & .33 & $.38 * *$ & $.66^{* * *}$ & $.61 * * *$ & 1.00 & & & & & & & & & \\
\hline 6. DASS-S & .17 & .23 & $.74 * * *$ & $.67 * * *$ & $.66^{* * *}$ & 1.00 & & & & & & & & \\
\hline $\begin{array}{l}\text { 7. PSAS- } \\
\operatorname{cog}\end{array}$ & .30 & .19 & $.77 * * *$ & $.67 * * *$ & $.63 * * *$ & $.71 * * *$ & 1.00 & & & & & & & \\
\hline $\begin{array}{l}\text { 8. PSAS- } \\
\text { som }\end{array}$ & .38 & $.29^{*}$ & $.62 * * *$ & $.54 * * *$ & $.76 * * *$ & $.56 * * *$ & $.71 * * *$ & 1.00 & & & & & & \\
\hline 9. CSD SE & .18 & -.15 & $.82 * * *$ & $-.57 * * *$ & $-.66 * * *$ & $-.50 * * *$ & $-.61 * * *$ & $-.59 * * *$ & 1.00 & & & & & \\
\hline $\begin{array}{l}\text { 10. CSD } \\
\text { SOL }\end{array}$ & .19 & -.01 & $.66^{* * *}$ & $.47 * *$ & $.41 * *$ & $.39 * *$ & $.62 * * *$ & $.41^{* *}$ & $-.72 * * *$ & 1.00 & & & & \\
\hline 11. Age & $-.49 *$ & .03 & $.37 * *$ & .20 & $.32 *$ & $.32 *$ & .23 & $.30 *$ & $-.41 * *$ & .25 & 1.00 & & & \\
\hline 12. Latency & .12 & .03 & .14 & .01 & .15 & .04 & .01 & .12 & -.17 & .08 & .07 & 1.00 & & \\
\hline 13. Amp. & .09 & -.04 & -.09 & .03 & .06 & -.11 & -.08 & .09 & -.06 & .01 & -.003 & -.01 & 1.00 & \\
\hline 14. Trauma & .39 & .28 & -.003 & .04 & .16 & .09 & .09 & .08 & -.13 & .12 & .04 & $.30 *$ & .01 & 1.00 \\
\hline
\end{tabular}

Note. RID $=$ Reactivity in the Dark. DDQ-Discomfort $=$ total score on the DDQ-Discomfort scale. DASS-D, $-\mathrm{A},-\mathrm{S}=$

Depression, Anxiety, Stress subscales on the DASS-21. PSAS-cog, -som = Cognitive and somatic subscales on the Pre-sleep Arousal Scale. CSD SE, SOL = Consensus Sleep Diary-measured Sleep Efficiency, Sleep Onset Latency. Latency $=$ Eyeblink latency in the second dark phase. Amp. = Eyeblink amplitude in the second dark phase. Trauma = past traumatic exposure (no PTSD diagnosis).

$$
*=\mathrm{p}<.05, * *=\mathrm{p}<.01, * * *=\mathrm{p}<.001
$$


Age analyses. To assess differences between GS and ID groups on subjective and objective FOD while controlling for age, age was entered as a covariate in two three-way mixed analyses of covariance (ANCOVAs) assessing group differences on eyeblink latency and amplitude across all four phases of the experiment. One participant in the GS group was not included in both analyses because age information was not completed in their battery of questionnaires. There was no main effect for age (small effect size; $r=.04$ ), and age did not significantly interact with block, phase, or group on eyeblink latency or eyeblink amplitude (see Tables 3 and 4 for statistics). 
Table 3

Three-way mixed analysis of variance assessing group differences between groups on eyeblink amplitude, with age as a covariate

\begin{tabular}{|c|c|c|}
\hline Variable & $F$ & $p$ \\
\hline \multicolumn{3}{|l|}{ Main } \\
\hline Block & 1.206 & .278 \\
\hline Phase & 0.368 & .547 \\
\hline Group & 0.078 & .782 \\
\hline Age & 1.486 & .229 \\
\hline \multicolumn{3}{|l|}{ Interaction } \\
\hline Block*Age & 2.779 & .278 \\
\hline Block*Group & 1.983 & .166 \\
\hline Phase*Age & 0.392 & .534 \\
\hline Phase*Group & 0.251 & .619 \\
\hline Block*Phase & 0.042 & .839 \\
\hline Block*Phase*Age & 0.440 & .510 \\
\hline Block*Phase*Group & 0.212 & .647 \\
\hline
\end{tabular}

Note . Phase $=$ lighting condition (light or dark). Degrees of freedom $(d f)$ for all effects: Hypothesis $d f=1$, Error $d f=46$. 
Table 4

Three-way mixed analysis of variance assessing differences between groups on eyeblink latency, with age as a covariate

\begin{tabular}{lcc}
\hline Variable & $F$ & $p$ \\
\hline Main & 0.020 & .888 \\
Block & 0.755 & .389 \\
Phase & 0.216 & .644 \\
Group & 0.827 & .368 \\
Age & & \\
Interaction & 0.053 & .819 \\
Block*Age & 0.461 & .501 \\
Block*Group & 1.225 & .274 \\
Phase*Age & 1.069 & .307 \\
Phase*Group & 0.858 & .359 \\
Block*Phase & 0.448 & .506 \\
Block*Phase*Age & 0.193 & .662 \\
Block*Phase*Group &
\end{tabular}

Note. Phase $=$ lighting condition (light or dark). Degrees of freedom $(d f)$ for all effects: Hypothesis $d f=1$, Error $d f=46$. 
In order to examine whether older and younger participants differed on the eyeblink variables, regardless of their group (sleeper status), a one-way ANOVA was conducted. Age was first made dichotomous via a median split, with participants 34 years of age and below categorized as "younger" and those older than 34 placed in the "older" group. Because age data was missing for one participant, the ANOVA was conducted with $n=49$ participants. Results confirmed that older and younger participants did not differ significantly on latency $(F(1,47)=$ $0.63, p=.43, r=0.11)$ or amplitude $(F(1,47)=0.18, p=.67, r=.06)$ of the eyeblink in the second dark phase of the experiment. Levene's test confirmed that the assumption of homogeneity of variances was not violated for latency $(p=.19)$ or amplitude $(p=.46)$. A oneway ANOVA with the dichotomous age variable as the between groups factor and the continuous DDQ-Discomfort score as the outcome variable was not significant, $F(1,46)=0.05$, $p=.83$. According to Cohen (1988) the effect size was small $(r=.03)$. The Levene's test indicated that the assumption of homogeneity of variances was not violated $(p=.39)$.

\section{Primary Analyses}

\section{Part 1: Quantitative.}

Subjective FOD. Forty-nine participants were included in the following chi-square analyses, as one participant had declined to complete the DDQ-Discomfort questionnaire and thus was missing data for this measure. A Pearson chi-square analysis was conducted to assess whether the ID group reported a subjectively greater proportion of discomfort in the dark compared to good sleepers on the single DDQ-Discomfort scale item that queried fear of "the dark." Eight individuals in the ID group and 9 in the GS group endorsed experiencing a degree of discomfort in the dark $\left(\chi^{2}(1)=.16, p=.67\right)$, while 17 individuals in the ID group and 15 GS reported no discomfort. The effect size was large according to Cohen's (1992) criteria $(d=.67)$. 
To assess whether group differences on the continuous DDQ-Discomfort score, a one-way ANOVA was conducted and showed that there were no differences between GS and ID, $F(1,47)$ $=0.57, p=.45$. The effect size for this analysis $(r=.11)$ was below the recommended minimum effect size (RMPE) for a significant effect (Ferguson, 2009), though it met criteria for a small effect according to Cohen (1992).

With respect to sex, although a greater proportion of females $(n=14)$ than males $(n=3)$ endorsed a mild or moderate discomfort in response to this item, the difference was not significant $\left(\chi^{2}(1)=2.67, p=.10\right)$. The size of this effect $(d=.48)$ was large according to Cohen (1992). There were however sex differences on the DDQ-Discomfort total score (as computed above), with a significantly greater proportion of females $(n=29)$ than males $(n=9)$ reporting FOD $\left(\chi^{2}(1)=6.19, p=.01\right)$; this was a large effect $(d=.76)$. However, a one-way ANOVA did not show sex differences on the continuous DDQ-Discomfort score, $F(1,47)=3.54, p=.07$. Cohen's $d$ was .55, a large effect size according to Cohen, and above the RMPE according to Ferguson (2009).

Objective FOD. To examine the startle indices of eyeblink magnitude and latency, two three-way mixed ANOVAs were conducted with block $(1,2)$ and phase (dark, light) as the within-groups factors and group (ID, GS) as the between-groups factor. Since preliminary analyses indicated that there was no effect for the order in which the lighting conditions were presented, and also no order-by-group interaction, eyeblink startle data was collapsed across order.

Eyeblink latency. Not every participant exhibited an eyeblink within the $20-120$ ms poststimulus threshold for startle. Indeed, though all 25 in the GS group exhibited a startle within this time period, only 22 from the ID group did. To ensure that these missing values (i.e., lack of 
a 'startle' in terms of reaction time) were included in final analyses, all missing values were coded as $121 \mathrm{~ms}$ (i.e., outside the window to count as a 'startle'). This transformation made it possible for all 50 participants to be included in the mean latency times in each of the four phases. Box's $M$ test was not significant $(p=.39)$ indicating that the assumption of homogeneity of covariance matrices was not violated. Because there were only two levels for each of the within-groups variables, the assumption of sphericity was not violated (Field, 2009). As such, Wilks' Lambda test statistics are reported (rather than the more conservative Pillai's Trace; see Tabachnick \& Fidell, 2001).

There was no significant main effect for the latency of the eyeblink between blocks ( $F(1$, $48)=1.18, p=.28$ ) nor phases (i.e., lighting conditions) $F(1,48)=0.39, p=.53$ ). The effect size was small $(r=.15)$ and also phase $(r=.08)$ according to Cohen's $(1988,1992)$ criteria. There was also no main effect for group $F(1,48)=1.54, p=.22$ (small effect size; $r=.18)$ There was no significant interaction between the block and group $(p=.53)$, phase and group ( $p=$ $.09)$, or phase and block $(p=.35)$ factors. There was no three-way interaction between block, phase, and group $(p=.91)$. Levene's test confirmed that the assumption of homogeneity of variances for the group variable (i.e., the between-groups factor) was not violated in any of the blocks or phases (all $p$-values $\geq .05$ ).

Eyeblink amplitude. Box's $M$ test was significant $(p=.007)$ indicating that the assumption that the within-group covariance matrices are equal was violated. Although Tabachnick and Fidell (2001) recommend a Box's $M$ significance cutoff of $p \leq .001$ before reporting Pillai's Trace statistics, Pillai's Trace is nonetheless reported in the interest of remaining conservative. There was no significant main effect for the magnitude of startle (i.e., amplitude of the eyeblink) between blocks $(F(1,48)=2.61, p=.11)$ nor phases, $(F(1,48)=$ 
$0.14, p=.71)$. The effect size was small-to-medium for block $(r=.23)$ and very small for phase $(r=.05)$. Additionally, there was no main effect for group on eyeblink amplitude $(F(1,48)=$ $0.12, p=.73)$. This effect size was small $(r=.05)$. There was no significant interaction effect for group by block $(F(1,48)=0.78, p=.38)$ nor group by phase $(F(1,48)=0.01, p=.92)$. There was however a significant interaction between the block and phase factors $(F(1,48)=$ $7.07, p=.01$ ), see Figure 2 for visual depiction of the interaction. There was no three-way interaction between block, phase, and group $(p=.41)$. Levene's test indicated that none of the dependent amplitude variables violated the assumption of homogeneity of variances $(p>.07)$. 


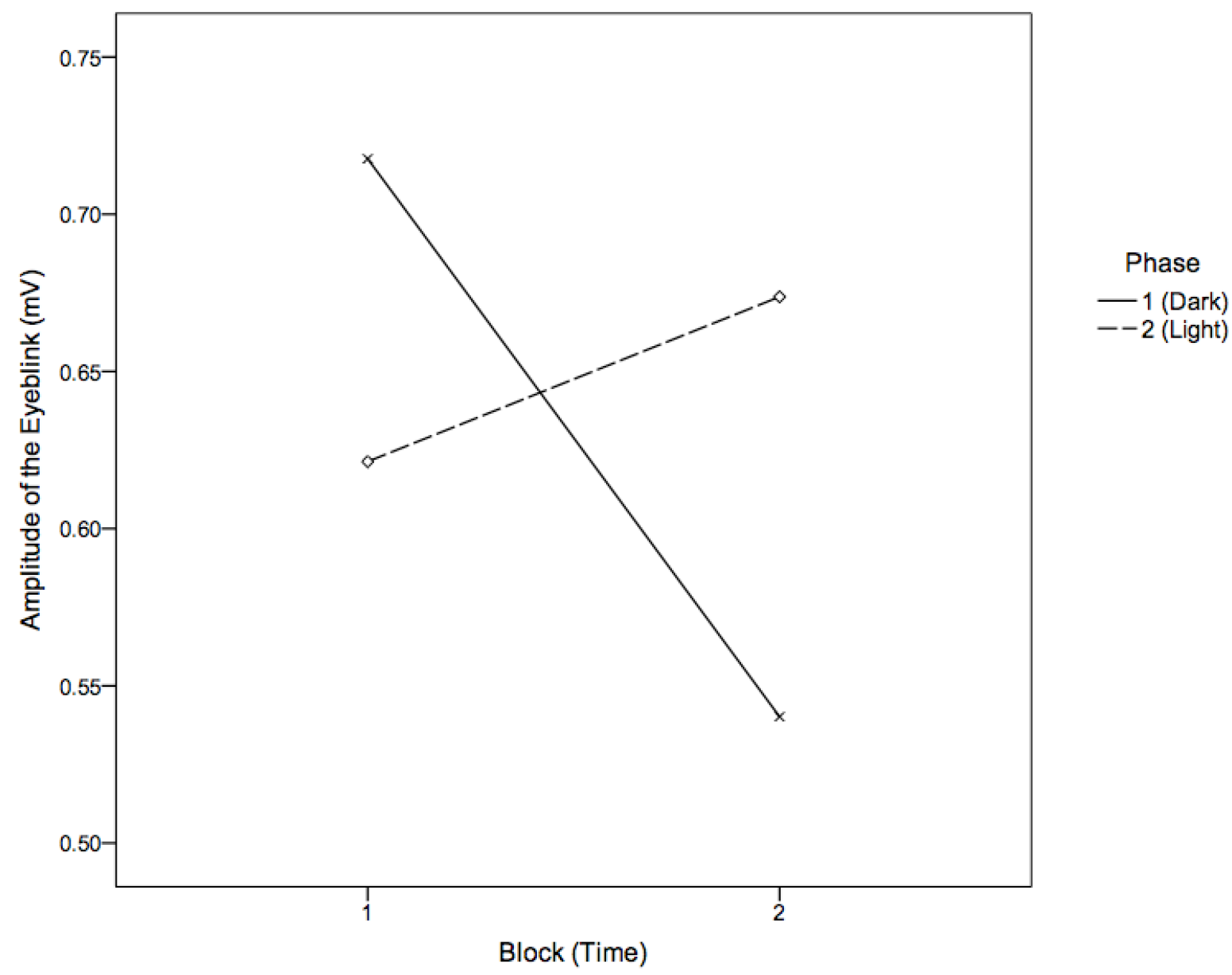

Figure 2. Graphical depiction of the interaction found between phase (dark, light) and block (1, 2) on eyeblink amplitude in the startle experiment. 
To follow up on the significant block-by-phase interaction found in the ANOVA on eyeblink amplitude, several paired samples $t$-tests were conducted. First, the differences between eyeblink amplitude in each phase over time were assessed. There was a significant difference between eyeblink amplitude in the dark in Block $1(M=.72, S D=.49)$ versus Block 2 $(M=.54, S D=.27), t(49)=3.11, p=.003$. The effect size $(d=.46)$ was medium according to Cohen (1992). There was no difference in amplitude in the light in Block 1 compared to Block $2, t(49)=-0.90, p=.38(d=.11)$. To test whether there were differences between mean eyeblink amplitude in the dark compared to the light in each block, two additional $t$-tests were conducted. There was no difference between amplitude of the eyeblink in the dark $(M=.72, S D=.49)$ compared to the light $(M=.62, S D=.39)$ in the first block, $t(49)=1.41, p=.17$. The effect size was small $(d=.22)$. In the second block, eyeblink amplitude was significantly different in the $\operatorname{dark}(M=.54, S D=.28)$ versus the light $(M=.67, S D=.56), t(49)=-2.12, p=.04$; the effect size was small $(d=.30)$.

Prepulse inhibition. To assess whether the prepulse stimuli (PP) affected the amplitude of the startle to the startle stimuli, the PP scores were compared across the dark and light phases. Commensurate with the procedure used to isolate eyeblink latency and amplitude after the acoustic stimulus pulses, PP stimuli were located visually and extracted using MATLAB. Unfortunately, a number of participants (30\%) had to be excluded from the prepulse analyses because visual detection of the PP stimuli was impossible. In these instances, the volume of the acoustic PPs was too low to visually discern the PPs separately from the background noise. Thus the following analyses utilized a sample of $n=35(\mathrm{ID}=17, \mathrm{GS}=18)$.

A two-way repeated measures ANOVA was conducted to examine differences between PP across all phases and blocks. The prepulse inhibition scores did not differ significantly across 
the light and dark conditions (phase main effect: $F(1,34)=.01, p=.92$ ). There was also no significant difference in PP scores between blocks (block main effect: $F(1,34)=.35, p=.56$. There was no significant interaction between phase and block $(F(1,34)=.01, p=.94)$. A threeway mixed ANOVA was conducted to evaluate whether PP scores differed between groups (GS, ID) across phases and blocks. There were no significant main effects (phase: $F(1,33)=.02, p=$ .90 , block: $F(1,33)=.32, p=.58)$, no significant interactions $(p=.22-.59)$, and no difference between groups on prepulse inhibition $(F(1,33)=0.15, p=.70)$.

Regression. A hierarchical multiple linear regression analysis was conducted to investigate potential predictors of the relationship between subjective insomnia severity and FOD. The sample for this analysis was $n=49$, due to the failure of one participant to complete the DDQ-Discomfort questionnaire. Using the enter method, the subjective and objective variables (DDQ-Discomfort total score and objective eyeblink amplitude and latency in the second dark phase) were entered in the first step. The hypothesized psychological variables (PSAS-cog, PSAS-som, DASS-D, DASS-A, DASS-S, past traumatic experience) were entered in the second step. The overall model was not significant at the first step $\left(R^{2}=.06, F(3,45)=\right.$ $.91, p=.44)$. The effect size $\left(f^{2}=0.06\right)$ was small according to Cohen (1988). The addition of the psychological variables in the second step significantly improved the predictive value of the model, $R^{2}=.83, F(6,39)=13.01, p<.0001$. The effect size was large $\left(f^{2}=4.88\right)$. Examination of the individual variables' beta values revealed that PSAS-cog was the only variable that was significantly related to the outcome variable of insomnia severity within this model. Table 4 presents the results of the regression analysis. Per guidelines for assessing for multicollinearity (Hair, Anderson, Tatham, \& Black, 1995; Tabachnick \& Fidell, 2001), Tolerance values were greater than 0.1 and Variance Inflation Factor (VIF) values were below 10.0 for all variables in 
the model. That the assumption of no multicollinearity amongst independent variables was not violated was confirmed via examination of the collinearity table; no values exceeded 0.8 (Field, 2009). Additionally, to assess whether a single participant may have affected the model as a whole, Cook's distance values were acquired and reviewed. No values were greater than 1.0, suggesting that no single case overly influenced the regression model (Cook \& Weisberg, 1982). 
Table 5

Hierarchical regression analysis predicting insomnia severity from subjective and objective FOD, depression, anxiety, and stress, pre-sleep arousal, and trauma incidence

\begin{tabular}{|c|c|c|c|c|}
\hline \multirow[b]{2}{*}{ Variable } & \multicolumn{4}{|c|}{ Insomnia Severity } \\
\hline & $\Delta R^{2}$ & $\beta^{+}$ & Partial $r$ & Partr \\
\hline $\begin{array}{l}\text { Step 1: } \\
\text { Predictor }\end{array}$ & .057 & & & \\
\hline Latency & & .096 & .098 & .096 \\
\hline Amplitude & & -.078 & -.079 & -.077 \\
\hline Discomfort & & .188 & .188 & .186 \\
\hline $\begin{array}{l}\text { Step 2: } \\
\text { Psychological }\end{array}$ & $.629 * *$ & & & \\
\hline DASS-D & & .062 & .055 & .031 \\
\hline DASS-A & & .059 & .048 & .027 \\
\hline DASS-S & & .226 & .212 & .121 \\
\hline PSAS-cog & & $.516^{*}$ & .456 & .287 \\
\hline PSAS-som & & .079 & .072 & .040 \\
\hline Trauma & & -.094 & -.144 & -.081 \\
\hline
\end{tabular}

Note. Latency $=$ mean eyeblink latency in Block 1, Phase 1 (dark). Amplitude $=$ mean eyeblink amplitude in Block 1, Phase 1 (dark). Discomfort = total score on the DDQ-Discomfort scale. DASS-D, A, $-\mathrm{S}=$ Depression, Anxiety, Stress subscales on the DASS-21. PSAS-cog, - som $=$ Cognitive and somatic subscales on the Pre-sleep Arousal Scale. Trauma = past traumatic exposure (no PTSD diagnosis).

+ Standardized regression coefficients are estimates from the regression models.

$* *=\mathrm{p}<.001, *=\mathrm{p}<.01$. 


\section{Part 2: Qualitative.}

Emergent content domains. In total, six key themes were identified based on the openended Post-Trial RID Interview questions (i.e., Part 1 of the interview). Table 6 presents the six content domains, brief descriptions of the domains, and a comprehensive list of examples of responses that fell within each theme. When describing their experiences in the dark, before bed, participants reported experiences that shared a number of similarities. Responses were grouped into thematically related clusters. The following content domains emerged from this analysis.

Production of sleep is contingent upon control. This theme is related to a desire to control the environment around oneself, perhaps as a proxy for controlling sleep itself. Responses included in this content domain tended to express a need to prepare in a detailed, perfectionistic manner for the sleep experience. This preparation often manifested as a nightly routine or ritual that took place in advance of a sleep attempt. Control was expressed variously as behaviours as well as attempts to control thoughts and feelings. The ultimate goal of exerting control via these varied rituals was the production of sleep. Participant quotes included:

I actually have to wear an eye mask now to sleep and I can't really I can't go to sleep without it so when I get to that point of winding down in my sleep cycle when I put the mask on it feels like I'm close to sleep and that feels really nice ... typically I sort of have my sleep kind of whatever weird you know sleep rituals down to a weird little art (70341) I wear like an eye mask to completely black everything out and have a white noise machine and I'm fine with it [the dark] (70271)

Anxious to perform the sleep. Within this domain, darkness cues bedtime, and bedtime means it is time for the individual to perform sleep. This theme is somewhat like performance 
anxiety, in that these individuals have, likely over time, learned that they are not good at performing sleep; this is an area that has been identified as problematic. As such, there is a feeling of increased pressure and stress related to whether the sleep will occur or not. As bedtime draws nearer, anxiety related to this performance increases. Participant quotes included: I don't mind the dark normally but I get anxious at night when it's time to fall asleep ... I guess I just feel the pressure of it being bedtime like I have to fall asleep it's not a fear of the dark it's a fear of the pressure of having to fall asleep (70326) Sometimes I just think about oh my god I'm not going to sleep (70287)

The mind is cued to take over. In this domain, the bedtime situation becomes an opportunity for the mind to have centre stage. The lights have been turned off, external stimuli have quieted, and there is the opportunity and time for the mind to think. It may be that this is the only time provided to process thoughts and feelings from that day, or an ample opportunity to engage in planning or strategizing about the next day. Depending on individual thinking styles, this type of thinking may be expressed as worrying or rumination. Thoughts within this domain are distinct from anxious thoughts about the impending sleep performance.

I think the darkness and especially bedtime for me it really brings kind of quiet things out in a way that at the same time wakes up my mind so it quiets out external factors but by removing the external influences and stimulants my mind is made awake and that keeps me up I seem to be more alert to my thoughts in the dark (70427)

When I'm in the dark and trying to go to sleep it seems like my mind just kicks into gear all the synapses are firing and I'm thinking about everything that happened during the day anything that could happen with other things in my life, any issues that are going on, 
and then trying to think of resolutions and action plans in terms of dealing with those situations whether they're large or minor and trying to think of ways to address or cope with them (70362)

Sometimes I rehash events for tomorrow. I think of things I have to do, sometimes get up and make a list. Generally just reviewing events of the day or things that are just concerning or stuff for the next day... or [I] think about life in general definitely things I shouldn't be thinking about when I'm trying to sleep (70287)

Increased caution, carefulness. This domain represents responses that expressed increased caution in the dark context. For some, this increase in caution was related to an overall feeling of vulnerability and decreased safety in the darkness. For others, the degree of caution expressed was dependent on context (e.g., a dark alley vs. at home). In general, the comments reflected non-extreme, rational responses to a change in lighting condition.

[The dark] doesn't bother me unless I'm in an alleyway on my own or underground parking lot with people I don't know right but even a power failure or anything it doesn't bother me if I'm at home I guess (70329) If I get up and go to the bathroom I'm a bit - not cautious - not afraid - I guess cautious $(70271)$

If I'm out walking I want it to be well lit for safety reasons (70408)

A feeling of tranquility and comfort. Within this domain, the dark is associated with positive emotions. Individuals expressed that they enjoyed the darkness particularly in the sleep 
context. In the sleep context, the darkness overall elicited positive, relaxing, even spiritual feelings and responses in this domain were related to a positive experience in the darkness. It's relaxing it's physically it feels good it feels relaxing to be in the dark (70449) I feel really content I'm just lying there in the dark and it's quiet (70365) [It's] tied to almost more spiritual feelings I don't want to say magical but possibilities seem more possible or real or something (70307) I really like the dark a lot for sleeping, like the darker the better ... it's very nice it's very soothing (70341)

Dislike, discomfort in, or aversion to the dark. The experience of the dark, generally across contexts, is aversive, uncomfortable, and unpleasant. Responses were related to a dislike of the dark that was separate from a rational increased sense of cautiousness and from the other content domains in general.

I don't like to be in the dark when there's absolutely no light at all it just feels very I don't know closed in but if there's some light then I'm fine (70374)

I want to say [I feel] terrified but I can walk in the dark I'd say more uncomfortable (70302) 
Table 6

The six emergent content domains, with descriptions and examples from participant responses.

\begin{tabular}{|c|c|}
\hline Theme & Description \\
\hline $\begin{array}{l}\text { 1. Production of } \\
\text { sleep is contingent } \\
\text { upon control. }\end{array}$ & $\begin{array}{l}\text { Control of the } \\
\text { environment is } \\
\text { necessary in order for } \\
\text { sleep to be } \\
\text { produced/initiated } \\
\text { when darkness falls. }\end{array}$ \\
\hline
\end{tabular}

Response examples

"I don't want to think about things that will upset me because I feel like it will prevent me from falling asleep so I try to count to 100" (70449).

I've tried the whole meditation thing ... or [I] just focus on something positive or anything like a trip coming up" (70432)

"I wear like an eye mask to completely black everything out and have a white noise machine and I'm fine with it [the dark]" (70271)

"In order to relax I need to write [thoughts] down because I feel like I'll forget for the next day" (70287)

I always sleep with a blanket over my ears so no noise will disturb me in the night I try to force myself to think of good things" (70302)

"I've trained my mind to think of me lying at the ocean listening to the waves" (70329)

"I actually have to wear an eye mask now to sleep and I can't really I can't go to sleep without it so when I get to that point of winding down in my sleep cycle when I put the mask on it feels like I'm close to sleep and that feels really nice... typically I sort of have my sleep kind of whatever weird you know sleep rituals down to a weird little art" (70341)

"let's see what else I'll get up have a bowl of cereal something to knock myself out" (70365)

"As related to insomnia if I can't stop my mind I take a hot bath but that becomes a bad thing where I worry if I don't take the bath I won't sleep (70378).

\section{Anxious to perform the sleep.}

Difficulty with sleep problems has, over time, caused increased pre-sleep stress and anxiety regarding whether the "sleep performance" will go well; this is cued by the dark, which is a signal for the sleep attempt to begin.
"Sometimes I just think about oh my god I'm not going to sleep" (70287)

"[I am feeling] anxieties about not sleeping especially if I have an interview or something tomorrow" (70287)

"I don't mind the dark normally but I get anxious at night when it's time to fall asleep so when I sometimes when I turn off the lights to go to sleep it makes me anxious... I guess I just feel the pressure of it being bedtime like I have to fall asleep it's not a fear of the dark it's a fear of the pressure of having to fall asleep" (70326)

"I also think about am I going to sleep at night am I 
going to get to sleep" (70374).

\section{The mind is cued to take over.}

With external stimuli quieted, the dark signals that this is now an opportunity for the mind to be able to think; the mind takes the centre stage, and stays on stage too long.
"Initially after the lights go out lately it's a feeling of anxiety whether I'm going to fall asleep at night" (70362).

"I'm frustrated because I'm not close to sleep" (70432)

“I'm wondering what's gonna happen and I don't know I kind of am constantly evaluating whether I'm actually sleepy and whether I'm going to sleep and what can I expect for the next hour or so" (70427)

"When I'm in the dark and trying to go to sleep it seems like my mind just kicks into gear all the synapses are firing and I'm thinking about everything that happened during the day anything that could happen with other things in my life, any issues that are going on, and then trying to think of resolutions and action plans in terms of dealing with those situations whether they're large or minor and trying to think of ways to address or cope with them" (70362)

"I think about the day I have a tendency to be one of these worry mongers I think about the past and what I've achieved and I get concerned if I haven't achieved what I wanted to do I start thinking about my life situation... that's when I start putting myself into a headspin sort of" (70365)

"I think the darkness and especially bedtime for me it really brings kind of quiet things out in a way that at the same time wakes up my mind so it quiets out external factors but by removing the external influences and stimulants my mind is made awake and that keeps me up I seem to be more alert to my thoughts in the dark" (70427)

"I find it's normally recapping of my day you know the days ahead anything that's stressing me out and it just becomes that repetitive pattern" (70432)

"Racing thoughts about the day's events" (70271)

Sometimes I rehash events for tomorrow. I think of things I have to do, sometimes get up and make a list. Generally just reviewing events of the day or things that are just concerning or stuff for the next day... or [I] think about life in general definitely things I shouldn't be thinking about when I'm trying to sleep" (70287)

"I think of negative thoughts... urban legends, movies I've watched, crime on the news" (70302)

\section{Increased caution, A rational response to "If I get up and go to the bathroom I'm a bit - not}


a condition in which

one has decreased visibility and as such, decreased safety; often expressed as dependent upon context. cautious - not afraid - I guess cautious" (70271)

"If I'm out walking I want it to be well lit for safety reasons" (70408).

"It's different than being in the light like I'm talking about pretty dark like can't find your way around" (70366).

"[It] doesn't bother me unless I'm in an alleyway on my own or underground parking lot with people I don't know right but even a power failure or anything it doesn't bother me if I'm at home I guess (70329).

"If I have to be out and its dark I do feel uncomfortable if I have to get somewhere if I have to be somewhere it's kind of weird for it to be dark because you never know what might be lurking" (70408).

"If I hear an odd noise or something then you get that feeling like maybe it's an intruder but I don't think it's tied directly to the dark I'd say maybe there's some vulnerability there you know because I'm laying in bed" (70432).

"I really like the dark a lot for sleeping ... but in a non-sleep environment it's not quite as comfortable, like in a tunnel or if the lights go out in an elevator I would feel pretty discombobulated (70341).

"It kind of goes back to the feeling of safety right like if I'm falling asleep I do prefer it to be dark but at the same time I need to know that my house is safe first" (70408).

\section{A feeling of tranquility and comfort.}

"I really like the dark a lot for sleeping, like the darker the better...it's very nice it's very soothing" (70341).

“On a good night I'm peaceful” (70378).

"It helps being in the dark. It's a help." (70327).

"I have a place out in the country I go to as often as possible and it's so dark I just love it" (70365).

"I feel really content I'm just lying there in the dark and it's quiet" (70365).

"[The dark is] relaxing and quiet" (70303). "[It's] tied to almost more spiritual feelings I don't want to say magical but possibilities seem more possible or real or something" (70307).

"It's relaxing" (70329).

"I generally have thoughts that are more focused on my body sort of mindful meditation thoughts like my legs are really heavy my back feels comfortable against the pillow" (70341). 
"It actually feels like a really safe and secure place so I generally associate it with positive feelings" (70341).

"Doesn't bother me ... I'm comfortable and I feel sleepy" (70346).

"I find the dark usually very comfortable (70362).

"I love it" (70365).

"It's relaxing it's physically it feels good it feels relaxing to be in the dark" (70449).

"When I'm at home in my own bed and stuff I find it very safe and comfortable (70449).

$\begin{array}{ll}\text { 6. Dislike of, } & \begin{array}{l}\text { Across contexts the } \\ \text { discomfort in, or }\end{array} \\ \text { aversion to the dark. } & \begin{array}{l}\text { is aversivence of the dark } \\ \text { uncomfortable, and } \\ \text { generally unpleasant. }\end{array}\end{array}$

"I want to say [I feel] terrified but I can walk in the dark I'd say more uncomfortable" (70302).

"Slightly uncomfortable um and I like to be able to see some sort of light" (70374).

"I'm not too fond of the dark but I can sleep through it fine" (70302).

"I don't like to be in the dark when there's absolutely no light at all it just feels very I don't know closed in but if there's some light then I'm fine" (70374).

"When I turn off the light and then I start to feel panicked at night and then I have to get up an turn the light back on and I feel myself calm down when the light is on" (70326).

"I'm not the biggest fan of like dark darkness when I'm trying to sleep" (70399).

"Being in the dark makes me feel weird" (70399).

"When I was a kid I had nightmares so being in the dark made me feel like I was in a nightmare (70399). 
Emerging pathways. In developing the content domains detailed above, two main theories emerged for delineating the relationship between the themes. Specifically, individuals appeared to experience the catalogued themes via (a) sleep performance pathway, or (b) trauma activation pathway (see Figure 3). The theme of "A feeling of tranquility and comfort" appeared to be separate from these pathways. Moreover, individuals may have endorsed specific themes without necessarily experiencing all stages of the theorized pathway.

Sleep performance pathway. Darkness triggers the onset of the sleep performance pathway. The dark cues the initiation of control behaviours in preparation for the eventual attempt to produce sleep. As an analogy, this first stage of the pathway is akin to setting up the stage in advance of a performance. The next stage in this pathway is the onset of anxiety related to the sleep performance. This anxiety is similar to pre-performance "jitters," with the difference being that the performance is sleep rather than acting, singing, or dancing. Finally, as the sleep attempt commences and the individual begins to doubt their performance abilities, the mind is cued and takes over as lead performer. At this final stage, thoughts take over for sleep and the individual likely begins to experience racing thoughts, worrying, or planning. It may be that the calm, quiet, carefully planned sleep environment provides a perfect opportunity for contemplation to occur. This may be especially true if there is less opportunity for such contemplation during the day (e.g., the individual avoids rumination via distraction techniques; the individual maintains a busy schedule with little time to relax and think during the day). Responses that mapped on to this pathway included:

I put blinds in my room just to make it as dark as possible ... um yeah if it's really dark I just hope I can fall asleep yeah I think about the day, I have a tendency to be one of these worry mongers I think about the past and what I've achieved and I get concerned if I 
haven't achieved what I wanted to I start thinking about my life situation, I guess I'm sort of one of these perfectionist people, caught up in details that's when I start putting myself into a head spin (70365)

I try to clear my head that is much easier said than done lately since this has been going on and it seems like once it starts there's this reaction of 'okay here we go again' and one things hops to the other and I feel like I'm getting anxious or agitated because there's so many thoughts going through my head combined with the frustration of feeling like it's going to be another night of hours of tossing and turning (70362) [I] sleep with like blackout curtains no lights on ... I don't know I mean it can become frustrating obviously when I'm not sleeping ... I find its normally recapping of my day you know the days ahead anything that's stressing me out and it just becomes that repetitive pattern and then that can also dovetail into the fact that I'm frustrated because I'm not close to sleep (70432)

Trauma activation pathway. Darkness is a cue for danger, which has been reinforced through previous traumatic event(s). The first stage involves a dislike of the experience of being in the dark. It may be that the darkness triggers trauma-related reminders directly. However it appears that the darkness often cues a second stage, specifically an evolutionarily adaptive sense of increased caution within a condition in which humans, as diurnal creatures, are naturally more vulnerable. In the dark, predators, obstacles, or other dangers cannot be seen and therefore cannot be planned for. This combination of physical vulnerability (i.e., lack of vision) and lack of awareness of one's surroundings means that one cannot protect oneself from these potential dangers. This in turn triggers the final stage of this pathway, wherein the mind is cued to take 
over. Though this final stage is similar to the way the mind is activated in the sleep performance theory, the way in which thoughts are activated is different in that there is a fraught attempt to regulate the thoughts and emotions that arise during this stage. This is likely in part due to tone these thoughts take; they are likely reminders of the trauma, and therefore, within this framework, ought to be avoided or controlled. Examples of responses that mapped onto this pathway included:

I don't know why I don't like it but it makes me anxious I'm one of those people that has racing thoughts when I'm trying to sleep and being in the dark makes me feel weird when I get really anxious my reaction is to turn on the light and that makes me feel I don't know better ... I feel like being able to see my surroundings makes me feel more grounded so I can calm down (70399) um I don't like to be in the dark when there's absolutely no light at all it just feels very I don't know closed in but if there's some light then I'm fine ... sometimes [I'm] thinking about things that have to be done tomorrow, and you know if there's something on my mind I'll go over you know over something that troubled me during the day sometimes if there was something (70374) I'm not too fond of the dark ... I think of negative thoughts (70302) 


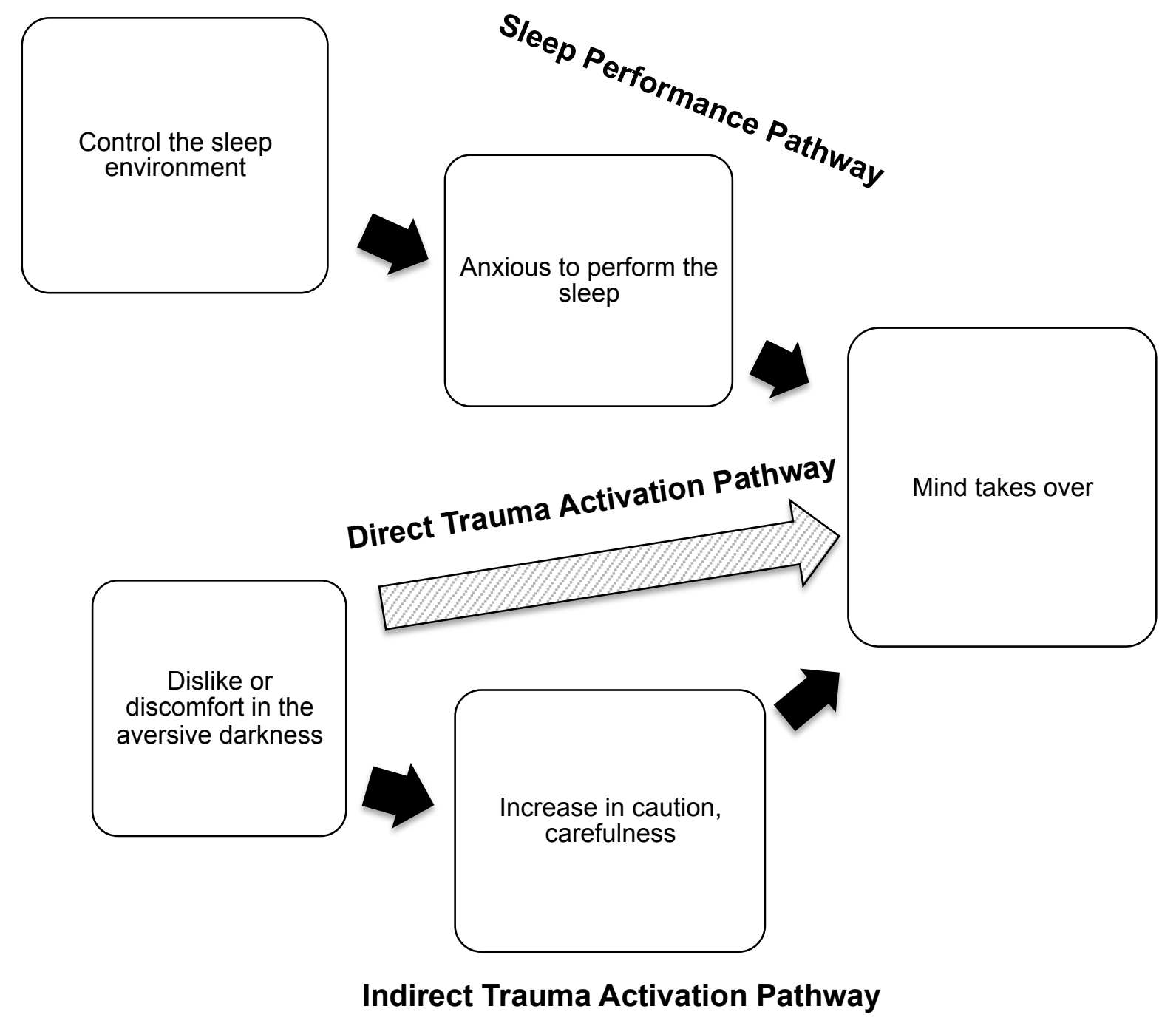

Figure 3. Emergent theories from thematic analysis of participant responses on the Post-Trial RID Interview: the Sleep Performance Pathway and the direct and the Trauma Activation Pathway. 


\section{Post Hoc Analyses}

In order to follow up on both the significant and the non-significant findings, a number of post-hoc analyses were conducted.

Since the preliminary chi-square analyses showed that a greater proportion of females endorsed an FOD on the overall DDQ-Discomfort score, a one-way ANOVA was conducted to assess for sex differences on the physiological startle in the dark variables (i.e., on eyeblink amplitude and eyeblink latency). Sex was the between-groups variable and eyeblink latency and amplitude were the dependent variables. Levene's test was not significant for amplitude, confirming that the assumption of homogeneity of variance was met. This assumption was however violated for eyeblink latency (Levene's statistic $=5.19, p=.03$ ). As such, for sex differences on latency, the more robust Brown-Forsythe $F$-ratio statistics are reported (Field, 2013). Results showed that females had a larger mean eyeblink amplitude in the second dark phase $(M=.57, S D=.27)$ compared to males $(M=.48, S D=.29)$, however this difference was not significant, $F(1,48)=0.95, p=.33$. Females also had a longer reaction time (eyeblink latency) in the second dark phase $(M=65, S D=25)$ compared to males $(M=57, S D=16)$, though the difference was not significant, Brown-Forsythe's $F(1,43.39)=1.89, p=.18$.

To assess whether age predicted insomnia severity beyond the predictive value of the FOD variables and the psychological variables, two hierarchical linear regressions were conducted with age in the first step and insomnia severity (ISI score) as the outcome variable. In the first regression, the original hypothesized FOD variables (DDQ-Discomfort score, and eyeblink amplitude and latency in the second dark phase) were entered into the second step. Because one participant had not completed age information, and one participant failed to complete the DDQ measure, this analysis was conducted with $N=48$ participants. The first step 
of the model was significant, $R^{2}=.14, F(1,46)=7.34, p=.009$, with age accounting for $1.4 \%$ of the variance in insomnia severity. The effect size $\left(f^{2}=0.16\right)$ was medium according to criteria (Cohen 1988). The addition of the subjective and objective FOD variables in the second step did not improve the model significantly $\left(R^{2}=.18, F(3,43)=0.66, p=.58\right)$. The effect size $\left(f^{2}=\right.$ 0.22) at this step was medium. For all variables, Tolerance and VIF values were within limits for excluding multicollinearity.

In the second regression, age was again entered in the first step, and the original hypothesized psychological and trauma variables (depression, anxiety, stress; PSAS-cog, PSASsom; trauma exposure) were added in the second step. Insomnia severity was again entered as the outcome variable. This analysis was conducted with 49 participants because one data point was missing for age. The model was significant in the first step, $R^{2}=.14, F(1,47)=7.68, p=$ .008 , with age accounting for $1.4 \%$ of the variance, as expected by results from the initial regression. The putative predisposing variables were entered into the second step to test whether they predicted insomnias severity over and above the effect of the FOD variables. The addition of the predisposing variables in the second step significantly improved the predictive value of the model, $R^{2}=.72, F(6,41)=13.79, p<.0001$, accounting for $5.75 \%$ of the variance in insomnia severity after controlling for age. At this step, the effect size $\left(f^{2}=2.57\right)$ was very large. Of the variables in the second step, only PSAS-cog shared a significant amount of variance with insomnia severity (part correlation $=.29, p=.001$ ) after controlling for the other variables in the model.

The DDQ-Discomfort scale was significantly correlated with a number of other variables of interest in the current study. Thus, to investigate predictors of subjective FOD on the DDQDiscomfort scale, a multiple linear regression $(n=49)$ with the physiologic objective measures 
of FOD (i.e., eyeblink latency and amplitude in the $2^{\text {nd }}$ dark phase) and the psychological variables (including the DASS subscales, the PSAS subscales, and trauma exposure) as predictors was conducted. The overall model was significant, $R^{2}=.45, F(8,40)=4.08, p=.001$, with the model accounting for $4.5 \%$ of the variance in subjective FOD on the DDQ-Discomfort scale. The effect size $\left(f^{2}=.82\right)$ was large. Examination of the beta coefficients indicated that both trauma exposure and cognitive pre-sleep arousal had a strong relationship with the outcome variable (see Table 7). 
Table 7

Hierarchical regression analysis predicting discomfort in the dark from eyeblink latency and amplitude, depression, anxiety, and stress, pre-sleep arousal, and trauma exposure.

\begin{tabular}{lcccccc}
\hline & \multicolumn{5}{c}{ Discomfort in the Dark } \\
\cline { 2 - 3 } Predictor Variables & \multicolumn{1}{c}{ Coefficient Statistics } & & \multicolumn{3}{c}{ Correlations } \\
\cline { 2 - 3 } \cline { 5 - 6 } Latency & $\beta^{+}$ & $p$ & & Zero-Order & Part & Partial \\
Amplitude & -0.239 & .072 & & .041 & -.281 & -.217 \\
DASS-D & -0.203 & .110 & & -.137 & -.250 & -.192 \\
DASS-A & 0.271 & .246 & & .302 & .183 & .138 \\
DASS-S & 0.358 & .158 & & .505 & .222 & .169 \\
PSAS-cog & -0.135 & .539 & & .336 & -.098 & -.073 \\
PSAS-som & $-0.411^{*}$ & .047 & & .222 & -.308 & -.241 \\
Trauma & 0.420 & .063 & .500 & .289 & .224 \\
\hline
\end{tabular}

Note. Latency $=$ mean eyeblink latency in Block 1, Phase 1 (dark). Amplitude $=$ mean eyeblink amplitude in Block 1, Phase 1 (dark). Discomfort = total score on the DDQ-Discomfort scale. DASS-D, A, $-\mathrm{S}=$ Depression, Anxiety, Stress subscales on the DASS-21. PSAS-cog, - som $=$ Cognitive and somatic subscales on the Pre-sleep Arousal Scale. Trauma = past traumatic exposure (no PTSD diagnosis). + Standardized regression coefficients are estimates from the regression models.

$*=\mathrm{p}<.05$. 
Because past experience of a trauma was significantly correlated with latency of the eyeblink in the second dark phase of the trial in the a priori correlations, a one-way ANOVA was conducted to assess differences in physiological startle (i.e., latency and amplitude of eyeblink in the $2^{\text {nd }}$ dark phase) between those that reported experiencing a trauma in the past $(n=$ 11 ) and those that reported no past trauma $(n=39)$. In terms of latency of eyeblink, there was a significant difference between those with past trauma $(M=76, S D=26)$ and those without past traumatic experience $(M=59, S D=21), F(1,48)=4.62, p=.04$. There was no difference in eyeblink amplitude across trauma groups, $F(1,48)=0.12, p=.75$. Levene's test confirmed that the assumption of homogeneity of variance was met for both analyses.

It was of interest to examine whether the subjective and objective FOD variables (eyeblink latency and amplitude, DDQ-Discomfort) were predictors of individuals' mean sleep onset latencies (pre-treatment). Though these variables were not significantly correlated in the $a$ priori Spearman's Rho analysis, it was hypothesized that the overall predictive value of the FOD variables might be stronger than the strength of the relationships between the individual variables. Thus, a multiple linear regression analysis $(n=49)$ was conducted with mean CSD SOL as the outcome variable and self-reported discomfort in the dark (DDQ-Discomfort score) and eyeblink latency and amplitude in the second dark phase entered as the predictor variables. The regression model was not significant, $R^{2}=.04, F(3,45)=0.56, p=.64$. The same analysis was conducted with CSD SE as the outcome variable, and was likewise non-significant, $R^{2}=$ $.03, F(3,45)=0.51, p=.68$.

Given that the Post-Trial RID Interview was designed for the current study, it was of interest to evaluate whether the second part of the interview, which assesses for frequency of thoughts and feelings prior to bed (e.g., feeling of a lack of safety), mapped on to the other 
objective and subjective measures of FOD in the current study (i.e., eyeblink amplitude and latency, and DDQ-Discomfort score). Because the Post-Trial RID Interview was only conducted with participants in the ID group, and interviews were not completed with three of the ID participants, this analysis was conducted with a sample size of 22. A multiple linear regression was conducted with part 2 of the Post-Trial RID Interview as the outcome variable and the FOD variables as the predictors. The model was significant, $R^{2}=.40, F(3,18)=4.03, p=.02$. The effect size was large $\left(f^{2}=0.67\right)$. Examination of the beta coefficients revealed that discomfort in the dark was the sole significant predictor of the frequency of FOD-related thoughts and feelings before bed (standardized $\beta=.65, t=3.47, p=.003$ ). 


\section{Discussion}

Contrary to hypotheses, good sleepers did not differ significantly from those with insomnia on objective startle indices in the dark or subjective FOD. The FOD indices (objective and subjective) were not significant predictors of insomnia severity. Cognitive pre-sleep arousal emerged a significant predictor of self-reported insomnia severity, even after controlling for age. Taken together, the eyeblink indices (amplitude and latency), mood variables (depression, anxiety, stress, pre-sleep arousal), and trauma exposure significantly predicted subjective discomfort in the dark; however, trauma exposure and cognitive pre-sleep arousal appeared to be the key predictors of discomfort in the dark. Further investigation of the trauma-FOD relationship revealed that those who had experienced a past trauma (of any kind) showed a significantly slower eyeblink reaction (latency) in the second dark phase compared to those with no trauma. Finally, qualitative analyses revealed two emergent pathways of participants' experiences in the dark: sleep performance and trauma activation.

Initial analyses of the relationships between the variables of interest revealed that the only variable that was significantly associated with eyeblink latency was trauma experience $(r=.30, p$ $<.05)$. This relationship was in the opposite direction than was expected; that is, presence of a past trauma was associated with longer eyeblink latency in the second dark phase, suggesting an increased startle in those without past trauma. Age was inversely associated with endorsement of pre-sleep thoughts and feelings related to FOD (e.g., feeling of a lack of safety before bed) on the Post-Trial RID Interview questions, suggesting that older age was associated with less frequency and younger age associated with greater frequency of FOD thoughts and feelings before bed. This relationship is interesting given that the sample in the current study included a wide age range, while Carney and colleagues (2013) employed a young adult, undergraduate 
sample and found different results. Given the discrepancy in findings between the two studies, it is possible that the broader the age range in the current sample accounted for some of the differing results found in the current study. However, subsequent analyses indicated that age was not a significant predictor of physiological startle (eyeblink latency or amplitude) in the second dark phase. Older age was significantly associated with greater insomnia severity, greater anxiety and stress, increased somatic pre-sleep arousal, and decreased sleep efficiency, all of which could be expected based on previous literature (for review see Ohayon, 2002).

Subjectively, participants with insomnia did not report greater fear of the dark than those with good sleep on either the single item that queried about the dark or on the DDQ-Discomfort total score. This result diverges from hypotheses, as well as past findings that showed a greater proportion of undergraduate students with poor sleep reported current FOD compared to those with good sleep (Carney et al., 2013). However, an examination of participants' total DDQDiscomfort subscale score revealed that a greater proportion of females scored in the range commensurate with FOD compared to males, suggesting there may be differences between sexes on self-reporting of discomfort in the dark. Since this sex difference was not found on the single item that queried about the dark, it may be that amongst adults, both males and females will tend not to say they would feel uncomfortable in the dark. However, when considering a variety of other experiences that take place in the darkness (e.g., blackouts, walking at night, tunnels), women were more likely to report feeling uncomfortable across these situations compared to men. Though an ANOVA revealed that this sex difference was not significant, in the current sample, it may be that in the larger population, females feel more comfortable expressing discomfort in certain situations (e.g., walking at night) compared to men, who may believe it socially desirable to deny discomfort across all situations. Women may also experience greater 
risk in situations such as walking at night, and thus experience and report greater fear. Such a theory may map on to situations in which participants considered it socially acceptable to report experiencing discomfort.

In contrast to hypotheses, there were no main effects for group (ID vs. GS), block (1 vs. 2), or phase (light vs. dark) on either eyeblink latency or eyeblink amplitude. There was no significant effect of group, phase, or block on eyeblink latency or amplitude after controlling for the effect of age. There were no interaction effects on eyeblink latency (i.e., reaction time), but one interaction effect was found on eyeblink amplitude. There was a significant two-way interaction between phase (dark vs. light) and block (first vs. second) on eyeblink amplitude of the startle reaction. That is, regardless of group (ID vs. GS), participants showed a significantly greater magnitude of startle (eyeblink amplitude) in the first dark phase compared to the second dark phase. As hypothesized, there was no such difference in the magnitude of the eyeblink between the first and second light phases. This suggests that in the dark, individuals demonstrated an increased startle initially, but habituated to the startle stimulus in the darkened condition over time. Additionally, although participants showed greater mean eyeblink amplitude in the dark compared to the light at the outset of the experiment (block 1), this difference was not statistically significant. However, over time (block 2), participants showed an increased magnitude of startle (eyeblink amplitude) in the light compared to the dark, and this difference was statistically significant. Thus, findings suggest that in the dark, all participants initially startled (exhibited a large magnitude of eyeblink) in response to the startle stimulus, but over time, there was habituation. In the light, there was no difference in eyeblink magnitude over time. 
The results from the startle paradigm experiment in the present study are both similar to and in contrast with past research. Findings from the current experiment are commensurate with Grillon and colleagues' (1997) findings that startle onset latency did not differ across lighting conditions. However, the present study's findings are in opposition to the recent study by Carney and colleagues (2012) that found a significant three-way interaction on eyeblink latency when comparing good and poor sleepers. Carney and colleagues found that those in the good sleeper group habituated over time (i.e., between block 1 and block 2) in the dark, while there was no significant change in the startle onset latency for those with poor sleep. In the current study, all participants habituated over time in the dark and did not show significant change over time in the light; there was no difference between groups.

It is possible that the expected differences between the GS and ID groups did not emerge because the sample included a wider range of ages. Although all participants exhibited a startle initially in the dark, the current sample demonstrated habituation over time, regardless of sleeper status. That is, it may be that as humans age, they become less physiologically reactive in the dark compared to the light condition; they become accustomed to or more comfortable in the darkened environment simply due to greater experience in this condition compared to younger individuals. It is possible, then, that the inclusion of older individuals in the current study diluted the group differences that were found previously in a younger, university-age sample (Carney et al., 2013). Any dark-specific sensitization amongst those in the ID group might have been isolated to the younger subset of participants. If this were the case, this subset would have been too small to have an identifiable effect on the eyeblink indices. Since the ID sample in the current study was $n=25$, it is unlikely that a dark-specific startle in a smaller subset of this group would emerge as significant. 
Results from the present study are also in contrast to past studies that found a greater magnitude of startle (eyeblink amplitude) in the dark compared to the light (Grillon et al., 1997; Carney et al., 2013). Although findings from the current study did show a significant decrease in startle in the dark over time in both GS and ID groups, results also showed that eyeblink amplitude was not significantly larger in the dark compared to the light in block 1, and in fact, amplitude was larger in the light than the dark condition in block 2. Grillon and colleagues (1997) similarly found that participants habituated to the startle stimulus in the dark, however unlike the current findings, they also found that startle magnitude was larger in the dark compared to the light. Further, Grillon and colleauges did not find a significant phase by block interaction as was found in the current study. Similarly, Carney and colleagues (2013) found a main effect for phase but no interaction effect on eyeblink amplitude. That is, these previous studies found that eyeblink magnitude decreased from block 1 to block 2 , but this decrease was not phase- (lighting condition) dependent.

One possible explanation for the differing results could be that participants in the current study found the onset of the light condition to be relatively more aversive than the dark condition. Indeed, it was noted that a number of participants remarked on the unpleasant experience of switching from the dark condition to the bright light condition. This makes sense given that the pupil of the eye would have to rapidly contract in response to a bright light, and this response would most likely coincide with a protective squint of the eye. It may be that the EOG electrodes picked up on this response and coded it as an eyeblink. However, the lightspecific "startle" found in the current study may have actually been a normative, protective physiological reaction in response to the onset of bright light. 
A key difference between these previous studies and the current study may be age. It is notable that both previous studies (Carney et al., 2013 \& Grillon et al., 1997) utilized university students in their samples, with mean ages of approximately 21 years (Grillon et al., 1997) and 22 years (Carney et al., 2013), while the current study included individuals with both clinical insomnia and good sleep with a mean age of 38 years (range: 18-71 years). Thus although there were no significant age-related results on the objective startle indices in the present study, consideration of age differences between the previous and present studies may be worthwhile in future analyses.

Neither subjective nor objective FOD measures (DDQ-Discomfort score, eyeblink latency, eyeblink amplitude) accounted for a significant proportion of the variance in insomnia severity. Instead, after controlling for the FOD variables, the psychological variables accounted for a very large amount (62.9\%) of the variance in insomnia severity. An examination of the individual contribution of each psychological variable revealed that cognitive pre-sleep arousal was the sole predictor that contributed uniquely to the model when the other variables were held constant. Thus while controlling for the other variables in the model, PSAS-cog shared $2.8 \%$ of the variance in insomnia severity. It is notable that the stress subscale of the DASS-21 shared $1.2 \%$ of the variance in insomnia severity, though the relationship between stress and the outcome variable was not significant within the model (i.e., with the other variables held constant). Since the association between PSAS-cog and insomnia severity was positive, it is clear that increased cognitive arousal during the sleep attempt is correlated with an increase in insomnia symptoms.

It is interesting that the FOD measures did not predict insomnia severity. It is clear that individuals experience heightened arousal in preparation for bedtime (Bonnet \& Arand, 1998). 
Indeed, a target of CBT for Insomnia is targeting the hyperarousal that many individuals report experiencing once they've turned off the lights in preparation for bed (Edinger \& Carney, 2015). If evidence suggests that bedtime preparation cues increased hyperarousal, it makes sense that a chief characteristic of this preparation - the condition of darkness - would cue such arousal. Arousal in the dark condition might translate to objective startle in the dark. Perhaps more significantly, it is surprising that individuals with insomnia did not report that their experience of the dark was at least somewhat uncomfortable at a higher rate than individuals with good sleep. If dark is a defining characteristic of sleep preparation, it rationally follows that individuals with insomnia would be less fond of this condition compared to those without sleep problems. It may be that older adults, as were included in the current study, are less receptive to considering the dark as a potentially frightening condition; it may be that older individuals consider discomfort in the dark to be childish.

Alternatively, individuals with insomnia frequently report a desire to control their environment, including the lighting in the room, in order to facilitate sleep onset. If behaviours such as wearing eye masks and putting blackout blinds on the windows are part of the pre-sleep ritual for some individuals with insomnia, it makes sense that these individuals would not subjectively report discomfort in this same situation. In other words, if implementing complete or near-complete darkness is a strategy, or safety behaviour, for facilitating sleep, it would not be helpful to think of the dark as an uncomfortable experience. For this group of individuals, to think about the darkness as uncomfortable in any way might induce anxiety about their ability to control the sleep environment and ultimately, anxiety about their ability to produce sleep. Thus, it would be "safer" for such individuals to believe, or at least report, low discomfort in the dark. 
Follow-up analyses showed that there were no sex differences on the objective startle variables (i.e., eyeblink amplitude and latency). However, results showed that age accounted for a significant proportion of the variance in insomnia severity (1.4\%). As age increased, so did insomnia severity, which is commensurate with past studies (e.g., Ancoli-Israel \& Roth, 1999; Ohayon, 1996; see Ohayon, 2002 for review). After controlling for age, the addition of the FOD variables in the second step did not improve the predictive ability of the regression model. Interestingly, a second analysis showed that after controlling for age, the addition of the psychological variables in the second step still improved the predictive value of the model overall. Cognitive pre-sleep arousal remained the variable that shared the most variance with insomnia severity after age was accounted for (while controlling for the other variables in the model). Thus it appears that cognitive arousal before bedtime is of greatest importance in predicting insomnia severity in the current model, regardless of participants' age. This finding highlights the importance of cognitive arousal in those with insomnia disorder, regardless of their age, and suggests that for those who are resistant to a behaviour focused insomnia treatment, targeting cognitive sources of arousal may be clinically beneficial.

Although trauma exposure was not a significant predictor of insomnia severity, it contributed to the prediction of subjective discomfort in the dark. Indeed, both trauma exposure and cognitive pre-sleep arousal emerged as significant predictors of subjective discomfort in the dark, suggesting that there may be a relationship between the extent to which individuals are aware of and report their discomfort in the dark and the presence of a past trauma and related arousal at bedtime. That is, greater pre-sleep cognitive arousal and presence of trauma exposure both increased the likelihood that individuals scored in the FOD range on the DDQ-Discomfort scale. The relation between these variables makes sense in light of research showing that 
heightened arousal before bed may be present in individuals with diagnosed with PTSD; for example, Pigeon and colleagues (2011) posited that hyperarousal may become a conditioned response to the bedroom in those with interpersonal violence-type trauma. Results from the current study would suggest that it may not be that the trauma is associated with insomnia symptoms directly, but rather that an experience of trauma functions to decrease the degree of comfort one feels in the dark, when visibility is low and vulnerability appears heightened. Indeed, past researchers have posited that this bedroom-specific condition may be a related to the experience of reduced ability to be vigilant; in other words, "sleep may be perceived as dangerous if it restricts the ability to monitor one's surrounding" (Pigeon et al., 2011, referencing Craske \& Tsao, 2005). This description in fact maps quite well onto the experience of being in the dark: one's ability to monitor, to engage in the hypervigilance that is a hallmark of PTSD symptomology, is reduced in the dark. It follows, then, that an individual with a trauma history may experience a simultaneous increase in vigilance and overall level of cognitive arousal when the lights go out at bedtime.

Extending this relationship between cognitive arousal, past trauma, and self-reported FOD, a theory of trauma activation emerged from analyses of responses from the ID group on the Post-Trial RID Interview. From the responses provided, a theoretical pathway was developed wherein the onset of darkness triggers an aversive reaction (or discomfort), which activates thinking via a familiar activation of the past traumatic experience. That is, cognitive arousal is cued by the darkness, via activation of the trauma pathway.

In the sleep performance pathway that emerged from the thematic analyses, individuals first attempt to take control of their sleep experience by "setting the stage" for performing highquality sleep. This setting of the stage then leads to, and may in fact contribute to, the onset of 
anxiety about the incumbent production of sleep, via anxious cognitions such as "am I going to get to sleep?" (70374). At this point, the heightened anxiety leads to pre-sleep cognitive arousal, which interferes with sleep onset. This pathway is supported by the strong correlations between DASS-S, DASS-A, and PSAS-cog. Though the current study did not include a measure of need for control specifically, past studies have found a relationship between need for control and stress levels (e.g., Averill, 1973; Nelson, Hammen, Daley, Dorli, \& Davila, 2001). Moreover, it makes sense that individuals experienced a degree of stress about their sleep problem that contributed to the onset of the enactment of this sleep performance pathway. Responses from individuals expressing such sentiments included use of phrases such as "the pressure of having to fall asleep" (70326); thus to reduce this feeling of stress and uncertainty, they engage in behaviours (e.g., eye masks, blackout blinds, blanket over ears) or attempt to enact cognitive processes (e.g., counting, imaginal relaxation techniques, meditation). Although the control may have been exerted over the environment, or even to an extent over cognitions, the high effort made to control the sleep performance in fact tends to make sleep worse (Harvey, 2002b; Hood, Carney, \& Harris, 2011).

In spite of the relationship between subjective FOD, heightened trauma, and pre-sleep cognitive arousal elucidated in the current study's results, these findings were not mirrored on the objective measures. Results indicated that regardless of sleeper status, those with past trauma exhibited a significantly slower startle onset latency compared to those with no trauma in the second dark phase of the experiment, and no difference on magnitude of the startle (i.e., eyeblink amplitude). However, given that the current study showed no expected differences on latency of the eyeblink after the startle stimulus presentation, it is possible that there is a spurious variable that has not been examined that has impacted the eyeblink latency effects. That is, it 
may be that the startle onset latency is only significantly faster in the dark in a subset of individuals across sleeper status, or that it is only a small subset of individuals with insomnia that exhibit a startle at all, and as such our study was underpowered to detect an effect in a small subset. Moreover, a past study found no differences between poor sleepers and good sleepers on eyeblink latency in the dark, except that good sleepers habituated to that condition over time (Carney et al., 2013). It may be that eyeblink latency is a less robust method of detecting startle response in dark and light conditions across individuals with good and poor sleep.

Nevertheless, it is interesting that there was a positive relationship between trauma and increased subjective FOD, but an inverse relationship between trauma and objective FOD. Specifically, not only did findings show that individuals with a history of trauma did not experience a greater magnitude of startle in the present study, but in fact results indicated that those with a past trauma experience exhibited a slower latency of startle compared to those with no trauma in the second dark phase of the study. A past study examined the magnitude of darkspecific startle in a sample that consisted of veterans of the Vietnam War with diagnosed PTSD compared to individuals with no PTSD (Grillon et al., 1998). Those who did not have PTSD included both civilians and war veterans. The findings from the current study are in opposition to this past research; Grillon and colleagues found that a) participants with PTSD exhibited an exaggerated startle response across all lighting conditions compared to individuals without PTSD, and b) participants that were war veterans exhibited a greater startle than those who had never experienced war, regardless of PTSD diagnosis (Grillon et al., 1998). That is, for those who were veterans of the war in Vietnam, both those who had PTSD and those with no PTSD diagnosis exhibited greater startle in the dark than did those who had no war experience. This 
suggests that the experience of trauma (e.g., war) may be sufficient to demonstrate a startle effect in dark conditions, regardless of whether or not PTSD develops.

The results of the current study are puzzling considering the findings of this previous study by Grillon and colleagues (1998). One possibility is that there could be substantive differences between individuals who have experienced traumas of various duration and severity, and that these differences may affect individual startle response. That is, as was presently hypothesized, and as the past study (Grillon et al., 1998) found, it may be that it is not diagnosis of PTSD that matters, but presence of a trauma. However, since the current findings did not show differences in the expected direction between those with and without trauma, as could have been expected based on the Grillon and colleagues study, it is possible that not all traumas are equal. Given the disparity between the results in the current study and the findings of Grillon and colleagues, it may be that the type of trauma is important in determining whether an individual will exhibit objective FOD (or endorse a subjective FOD). The importance of differences between trauma types and experiences is mirrored in the PTSD literature (e.g., Weathers \& Keane, 2007), suggesting that trauma type may play a role in later symptom manifestation. It makes sense, then, that type of trauma might matter in terms of dark-specific FOD reactions. For example, individuals who have gone through a war - an experience that is likely to have been protracted and included multiple traumas - may be experience the dark quite differently compared to individuals who have experienced single event exposure to trauma, such as a car accident or other potentially dangerous situations. The experience of participating in a war has the potential to contain innumerable traumas; a single trauma may manifest differently in terms of how a person experiences and reacts in the dark. 
If the chronicity, severity, and/or number of traumas impacts the development of a startle reaction in dark conditions, this would explain why Grillon and colleagues found that the war veterans in their sample exhibited greater startle in the dark compared to civilians (i.e., individuals with no war experience), and why individuals in the current study, with a wide-range of trauma experience ranging in severity and duration, did not. It would be interesting to focus on differentiating between traumatic experiences in future research in order to tease apart potential differences on dark-specific startle.

Another hypothesis that could be explored is that sleep problems might mediate the relationship between trauma and dark-specific startle. Although the current study sampled individuals who had experienced trauma in both the GS and ID groups, individuals with a trauma history comprised only a small proportion of the total sample $(n=11)$, with six in the ID group and five in the GS group; thus between-group, trauma-specific analyses in the present study would have been underpowered. An important next step will be to replicate this study in a larger sample of individuals with a history of trauma, across GS and poor sleeper groups.

It is also conceivable that a relationship between FOD and trauma is specific only to subjective measures of FOD. It may be that the experience of a past trauma leads individuals to experience heightened vigilance and arousal in situations that bear to potential to increase one's vulnerability. Over time, though PTSD may not develop, this experience may become conditioned in the dark, but it is possible that one's awareness of increased vulnerability in the dark does not disappear. That is, individuals with past trauma may possess a belief that the dark is inherently less safe than the light, and they are aware of this belief and in turn report a greater degree discomfort in the dark. If this is the case, perhaps for non-PTSD, single event trauma, the objective startle response is not present. Indeed, given the current findings, perhaps this 
awareness of a heightened vulnerability or discomfort in the darkness increases resilience to startle, and this is why individuals with trauma were found to react more slowly than the notrauma group in the second dark phase of the startle experiment.

The current study was designed to replicate, in a clinical sample, previous research that found that individuals with poor sleep both subjectively reported and objectively demonstrated greater FOD than those with good sleep (Carney et al., 2013). Although a priori power analyses suggested a minimum sample size of approximately 50 participants, the study by Carney and colleagues had a sample size of $N=108$, with more participants in the GS group than the poor sleeper group. To detect a medium effect size on the objective startle analyses (as was found in the Carney et al. study), Cohen (1992) recommended a sample size of 52 participants per group for an ANOVA involving three variables, as was the case in the present study. Thus according to Cohen the current study may have been underpowered. Future research should recruit a minimum of 50 participants per group to assess whether differences emerge in a larger, betterpowered sample.

Additionally, although both studies utilized MATLAB software to extract the data into Microsoft Excel files (which were then imported to SPSS), Carney and colleagues (2013) utilized monitoring technology Grass Technologies (a product group of Astro-Med, Inc.), while the current study used a Biopac physiological monitoring system. Although a similar pattern to the previous study ought to have emerged in the eyeblink data from the current pattern, it is possible that the differing measurement systems are responsible for a portion of the variance in the results between the two studies. In the previous study, for example, the two EOG electrodes measuring eyeblink activity were placed on the outer edge of the brow bone above the right eye, and at the outer, lower edge of the left eye. For the current study, both electrodes were placed at 
the outer edge and just below the right eye, as per Biopac guidelines (Blumenthal et al., 2005). If this latter electrode location increased the sensitivity with which eyeblink amplitude was measured compared to the previous system, this might explain the increased magnitude of startle that some participants exhibited in the light condition (i.e., they may squinted in response to the light, rather than reacted with a startle). Replication in a larger sample, with similar physiological measurement systems, is needed in order to ascertain whether the significant and non-significant results from the current study represent the true experiences of the two populations (GS and ID).

Given the sleep performance pathway revealed by the qualitative results, and supported by the correlational quantitative results, it would be interesting to investigate the associations between control over the sleep environment, anxiety related to sleep, and cognitive arousal at bedtime in those with insomnia in further depth. Whether these individuals first experienced sleep problems or first experienced a need to control their surrounding environment would be an intriguing question for future research. That is, did a need to control one's sleep schedule, pattern, duration, depth lead to sleep problems (sleep effort causing insomnia), or did a chronic problem with sleep lead to a desire to attempt to control it, in order to set oneself up for the best possible night?

The question of chronicity is not only of interest as it pertains to the theory of a sleep performance pathway leading to cognitive pre-sleep arousal, but also as it pertains to the objective indices of startle in the current study. From the outset of this study it was hypothesized that it may be a subgroup of individuals with insomnia that experience greater objective startle in the dark than good sleepers. If this were the case, this subgroup would actually differ from the other members of the ID group, meaning that the sample sizes were likely unbalanced. 
Depending on the size of this theoretical subgroup, it is entirely possible that the current study did not have adequate power to detect differences between this subgroup and the other participants.

The current study design differs from previous studies (Carney et al., 2013; Grillon et al., 1991, 1997) in a number of ways, including that participants in the current study were older and ranged more widely in age than the participants in previous studies. In addition, participants were stratified based on whether they met RDC for good sleep or insomnia disorder. Participants in the current study were community members who volunteered as research participants; those in the ID group volunteered to participate in four one-hour sessions of CBT-I therapy over a twomonth period after the current experiment's completion. All participants agreed to maintain participation in the study for a one-year period that includes monthly follow-up phone calls. That is, it may be that the participants in the current study differed significantly from the general population of individuals with clinical insomnia and those with good sleep. An ideal method of extending this research in future studies would be to complete this FOD startle experiment as part of a routine physical assessment at the time that an insomnia-specific sleep complaint is made at a primary care facility. In lieu of such a sweeping study, future research should focus on gathering specific data on the chronicity of insomnia disorder as well as specifics related to trauma duration, severity, and number of traumatic events experienced. Differences on these variables may yield differential dark-specific startle responses in future research. In addition, variables such as the presence of sleep-related effortful (control) behaviours and anxiety should be incorporated into future studies in a larger sample of adults, ranging widely in age, sex, and socio-economic status. The two FOD pathways posited in the current study will be useful in 
informing future work, in particular in designing studies examining the development of conditioned cognitive arousal in the dark, before bedtime.

In summary, findings from this study suggest that in an adult population that includes a wide range of ages, individuals with insomnia disorder do not differ from those with good sleep on objective measures of dark-specific startle. Subjectively, females reported greater FOD than males, suggesting that there may be sex differences in terms of willingness to report discomfort or experience discomfort in dark situations. Pre-sleep cognitive arousal emerged as an important factor in predicting insomnia severity and also in predicting subjective discomfort in the dark. Past experience of trauma was also strongly related to self-report of discomfort in the dark. Analysis of participant interview responses revealed two potential key pathways to cognitive arousal in the dark: a sleep performance pathway, and a trauma activation pathway. Although the current study did not provide evidence for the presence of a specific FOD construct in individuals with insomnia that is separate from pre-sleep arousal, findings have raised a number of questions about the way in which adults experience the dark. Future studies should focus on trauma history and pre-sleep cognitive arousal as factors that may be particularly important in the symptom manifestation and ultimately, clinical treatment of individuals who struggle with insomnia at night, in the dark, while waiting for sleep. 
ID:

Group: CUR PAST GS

Date:

\section{Appendix A}

\section{Semi-Structured Post-Trial RID Interview}

Part of the purpose of this study is to examine the nature of people's experiences in the dark.

1. In general, when you're in the dark, what is that experience like for you?

2. Before you go to sleep, when you are in the dark, what is your experience like for you?

3. What kind of thoughts go through your head when you are in the dark and going to sleep?

4. How do you feel when you are in the dark?

(if unclear) What kind of emotions do you experience when you are in the dark?

5. Are there any things you do because of how you're thinking and feeling during these times?

(if unclear) Do you do anything to help manage or control these thoughts and feelings? 
As researchers, we are trying to understand what it might be about the dark situation that heightens people's reactivity. Based on existing research, there are some ideas as to what might be behind this phenomenon. Can you tell us whether you experience any of the following, more specific thoughts or feelings when you are in the dark?

1. Lack of safety?

If so:

- On a scale of 1 to 10 , with 10 being very frequently, how frequently do you experience this thought before bed?

\begin{tabular}{|l|l|llllllllll|} 
YES & NO & 1 & 2 & 3 & 4 & 5 & 6 & 7 & 8 & 9 & 10 \\
\hline
\end{tabular}

2. Fear of an intruder or break-in?

If so:

- On a scale of 1 to 10 , with 10 being very frequently, how frequently do you experience this thought before bed?

\begin{tabular}{|l|l|llllllllll|}
\hline YES & NO & 1 & 2 & 3 & 4 & 5 & 6 & 7 & 8 & 9 & 10 \\
\hline
\end{tabular}

3. Feeling of vulnerability?

If so:

- On a scale of 1 to 10 , with 10 being very frequently, how frequently do you experience this thought before bed?

\begin{tabular}{|l|l|llllllllll|}
\hline YES & NO & 1 & 2 & 3 & 4 & 5 & 6 & 7 & 8 & 9 & 10 \\
\hline
\end{tabular}

4. Feeling of uncertainty?

If so:

- On a scale of 1 to 10 , with 10 being very frequently, how frequently do you experience this thought before bed?

\begin{tabular}{|l|l|llllllllll|}
\hline YES & NO & 1 & 2 & 3 & 4 & 5 & 6 & 7 & 8 & 9 & 10 \\
\hline
\end{tabular}

6. Is there anything we've missed here? Anything else you'd like to add about your experiences in the dark?

Participant reviewed responses for accuracy? ___ Yes ___ No Notes: 


\section{References}

Ancoli-Israel, S. \& Roth, T. (1999). Characteristics of insomnia in the United States: Results of the 1991 National Sleep Foundation Survey. I. Sleep(Suppl. 2), 347-353.

Antony, M. M., Bieling, P. J., Cox, B. J., Enns, M. W., \& Swinson, R. P. (1998). Psychometric properties of the 42-item and 21-item versions of the Depression Anxiety Stress Scales in clinical groups and a community sample. Psychological Assessment, 10, 176-181. http://dx.doi.org/10.1037//1040-3590.10.2.176

Averill, J. R. (1973). Personal control over aversive stimuli and its relationship to stress. Psychological Bulletin, 80, 286-303. http://dx.doi.org/10.1037/h0034845

Babson, K. A., \& Feldner, M. T. (2010). Temporal relations between sleep problems and both traumatic event exposure and PTSD: A critical review of the empirical literature. Journal of Anxiety Disorders, 24, 1-15. http://dx.doi.org/10.1016/j.janxdis.2009.08.002

Balaban, M. T., \& Taussig, H. N. (1994). Salience of fear/threat in the affective modulation of the human startle blink. Biological Psychology, 38, 117-131.

Bastien, C. H., Vallieres, A., \& Morin, C. M. (2001). Validation of the Insomnia Severity Index as an outcome measure for insomnia research. Sleep Medicine Reviews, 2, 297-307. http://dx.doi.org/10.1016/s1389-9457(00)00065-4

Blumenthal, T. D., Cuthbert, B. N., Filion, D. L., Hackley, S., Lipp, O. V., \& Van Boxtel, A. (2005). Committee report: Guidelines for human startle eyeblink electromyographic studies. Psychophysiology, 42, 1-15. http://dx.doi.org/10.1111/j.1469-8986.2005.00271.x

Bonnet, M. H., \& Arand, D. L. (1997). Hyperarousal and insomnia. Sleep Medicine Reviews, 1, 97-108. doi:10.1016/S1087-0792(97)90012-5 
Borkan, J. (1999). Immersion/Crystallization. In B. F. Crabtree and W. L. Miller (Eds.), Doing Qualitative Research (2nd Ed., 179-194). Thousand Oaks, CA: Sage Publications.

Bradley, M. M., Cuthbert, B. N., \& Lang, P. J. (1996). Lateralized startle probes in the study of emotion. Psychophysiology, 33, 156-161.

Braun, V. \& Clarke, V. (2006). Using thematic analysis in psychology. Qualitative Research in Psychology, 3, 77-101. http://dx.doi.org/10.1191/1478088706qp063oa

Brown, T. A., Chorpita, B. F., Korotitsch, W., \& Barlow, D. H. (1997). Psychometric properties of the Depression Anxiety Stress Scales (DASS) in clinical samples. Behaviour Research and Therapy, 35, 79-89. http://dx.doi.org/10.1016/s0005-7967(96)00068-x

Buysse, D. J., Ancoli-Israel, S., Edinger, J. D., Lichstein, K. L., \& Morin, C. M. (2006). Recommendations for a standard research assessment of insomnia. Sleep, 29, 1155-1173.

Carney, C. E., Buysse, D. J., Ancoli-Israel, S., Edinger, J. D., Krystal, A. D., Lichstein, K. L., \& Morin, C. M. (2011). The Consensus Sleep Diary: Standardizing prospective sleep selfmonitoring. Sleep, 35, 287-302. http://dx.doi.org/10.5665/sleep.1642

Carney, C. E., Edinger, J. D., Olsen, M. K., Stechuchak, K. M., Krystal, A. D., \& Wyatt, J. K. (2008). Inter-rater reliability for insomnia diagnoses derived from the Duke Structured Interview for Sleep Disorders. Sleep(Suppl.), 31, A250.

Carney, C. E., Moss, T. G., Atwood, M. E., Crowe, B. M., \& Andrews, A. J. (2013). Are poor sleepers afraid of the dark? A preliminary investigation. Journal of Experimental Psychopathology, 1-12. http://dx.doi.org/10.5127/jep.032312

Clara, I. P., Cox, B. J., \& Enns, M. W. (2001). Confirmatory factor analysis of the DepressionAnxiety-Stress Scales in depressed and anxious patients. Journal of Psychopathology and Behavioral Assessment, 23, 61-67. doi:10.1023/A:1011095624717 
Cohen, J. (1992). A power primer. Psychological Bulletin, 112, 155-159. http://dx.doi.org/10.1037//0033-2909.112.1.155

Cohen, J. (1988). Statistical power analysis for the behavioral sciences (2nd ed.). Hillsdale, NJ: Erlbaum.

Cook, R. D., \& Weisberg, S. (1982). Residuals and influence in regression. New York: Chapman \& Hall.

Craske, M. G., \& Tsao, J. C. I. (2005). Assessment and treatment of nocturnal panic attacks. Sleep Medicine Reviews, 9, 173-184. http://dx.doi.org/10.1016/j.smrv.2004.11.003

Daley, M., Morin, C. M., LeBlanc, M., Grégoire, J.-P., \& Savard, J. (2009). The economic burden of insomnia: Direct and indirect costs for individuals with insomnia syndrome, insomnia symptoms, and good sleepers. Sleep, 32, 55-64. http://dx.doi.org/10.1016/s1073$5437(10) 79480-8$

Davis, M. (1974). Sensitization of the rat startle response by noise. Journal of Comparative and Physiological Psychology, 87, 571-581. http://dx.doi.org/10.1037/h0036985

Davis, M. (1992). The role of the amygdala in fear-potentiated startle: Implications for animal models of anxiety. Trends in Pharmacological Sciences, 13, 35-

41. http://dx.doi.org/10.1016/0165-6147(92)90014-w

DiCicco-Bloom, B., \& Crabtree, B. F. (2006). The qualitative research interview. Medical Education, 40, 314-321. http://dx.doi.org/10.1111/j.1365-2929.2006.02418.x

Edinger, J. D., Bonnet, M. H., Bootzin, R. R., Doghramji, K., Dorsey, C. M., Espie, C. A., ... \& Stepanski, E. J. (2004). Derivation of research diagnostic criteria for insomnia: Report of an American Academy of Sleep Medicine Work Group. Sleep, 27, 1567-1596. 
Edinger, J. D., \& Carney, C. E. (2015). Overcoming insomnia: A cognitive-behavioral therapy approach ( $2^{\text {nd }}$ ed.). New York: Oxford University Press. http://dx.doi.org/10.1016/j.cpr.2005.04.003

Edinger, J. D., \& Means, M. K. (2005). Cognitive--behavioral therapy for primary insomnia. Clinical Psychology Review, 25, 539-558.

Edinger, J., Kirby, A., Lineberger, M., Loiselle, M., Wohlgemuth, W., \& Means, M. (2004). The Duke Structured Interview for Sleep Disorders. Duke University Medical Center.

Edinger, J. D., Olsen, M. K., Stechuchak, K. M., Carney, C. E., Chiang, A., Krystal, A. D., ... \& Radtke, R. A. (2009). Reliability and validity of the Duke Structured Interview for Sleep Disorders for Insomnia Screening. Sleep, 32(Suppl.) A265.

Edinger, J. D., Wohlgemuth, W. K., Radtke, R. A., Marsh, G. R., \& Quillian, R. E. (2001). Cognitive behavioral therapy for treatment of chronic primary insomnia: A randomized controlled trial. The Journal of the American Medical Association, 285, 18561864. http://dx.doi.org/10.1001/jama.285.14.1856

Eysenck, H. J. (1965). Extraversion and the acquisition of eyeblink and GSR conditioned responses. Psychological Bulletin, 63, 258-270. http://dx.doi.org/10.1037/h0021921

Ferguson, C. J. (2009). An effect size primer: A guide for clinicians and researchers.

Professional Psychology: Research and Practice, 40, 532-538. http://dx.doi.org/10.1037/a0015808

Field, A. (2009). Discovering statistics using IBM SPSS Statistics ( $3^{\text {rd }}$ ed.). London: Sage. Field, A. (2013). Discovering statistics using IBM SPSS Statistics: And sex and drugs and rock ' $n$ ' roll ( $4^{\text {th }}$ ed. $)$. London: Sage. 
First, M. B., Spitzer, R. L., Gibbon, M., \& Williams, J. B. (2002). Structured clinical interview for DSM-IV-TR axis I disorders, research version, patient edition. New York: Biometrics Research, New York State Psychiatric Institute.

Freedman, R. R., \& Sattler, H. L. (1982). Physiological and psychological factors in sleep-onset insomnia. Journal of Abnormal Psychology, 91(5), 380389. http://dx.doi.org/10.1037//0021-843x.91.5.380

Fullerton, P. (2006). The economic impact of insomnia in managed care: A clearer picture emerges. The American Journal of Managed Care, 12(Suppl. 8), 246-252.

Gordon, J., King, N., Gullone, E., Muris, P., \& Ollendick, T. H. (2007). Nighttime fears of children and adolescents: Frequency, content, severity, harm expectations, disclosure, and coping behaviours. Behaviour Research and Therapy, 45, 2464-

2472. http://dx.doi.org/10.1016/j.brat.2007.03.013

Grillon, C., Ameli, R., Woods, S. W., Merikangas, K., \& Davis, M. (1991). Fear-potentiated startle in humans: Effects of anticipatory anxiety on the acoustic blink reflex. Psychophysiology, 28, 588-595. http://dx.doi.org/10.1111/j.1469-8986.1991.tb01999.x

Grillon, C., Morgan, C. A., Davis, M., \& Southwick, S. M. (1998). Effect of darkness on acoustic startle in Vietnam veterans with PTSD. American Journal of Psychiatry, 155, 812817. http://dx.doi.org/10.1016/s0006-3223(98)00034-1

Grillon, C., Pellowski, M., Merikangas, K. R., \& Davis, M. (1997). Darkness facilitates the acoustic startle reflex in humans. Biological Psychiatry, 42, 453460. http://dx.doi.org/10.1016/s0006-3223(96)00466-0

Hair, J. F., Anderson, R. E., Tatham, R. L., \& Black, W. C. (1995). Multivariate data analysis ( $3^{\text {rd }}$ ed.). New York: Macmillan. 
Harvey, A. G. (2002a). A cognitive model of insomnia. Behaviour Research and Therapy, 40, 869-893. http://dx.doi.org/10.1016/s0005-7967(01)00061-4

Harvey, A. G. (2002b). Identifying safety behaviors in insomnia. Journal of Nervous and Mental Disease, 190, 16-21. http://dx.doi.org/10.1097/00005053-200201000-00004

Hayes, A. F., \& Krippendorff, K. (2007). Answering the call for a standard reliability measure for coding data. Communication Methods and Measures, 1, 7789. http://dx.doi.org/10.1080/19312450709336664

Hood, H. K., Carney, C. E., \& Harris, A. L. (2011). Rethinking safety behaviours in insomnia: Examining the perceived utility of sleep-related safety behaviours. Behavior Therapy, 42, 644-654. http://dx.doi.org/10.1016/j.beth.2011.02.004

IBM Corp. Released 2011. IBM SPSS Statistics for Windows, Version 20.0. Armonk, NY: IBM Corp.

Jacobs, G. D., Pace-Schott, E. F., Stickgold, R., \& Otto, M .W. (2004). Cognitive behavior therapy and pharmacotherapy for insomnia: A randomized controlled trial and direct comparison. Archives of internal medicine, 164, 18881896. http://dx.doi.org/10.1001/archinte.164.17.1888

Lang, P. J., Davis, M., \& Ohman, A. (2000). Fear and anxiety: Animal models and human psychophysiology. Journal of Affective Disorders, 61, 137-

159. http://dx.doi.org/10.1016/s0165-0327(00)00343-8

LeBlanc, M., Merette, C., Savard, J., Ivers, H., Baillargeon, L., \& Morin, C. M. (2009). Incidence and risk factors of insomnia in a population-based sample. Sleep, 32, 1027-1037. 
Leger, D., Guilleminault, C., Dreyfus, J. P., \& Delahaye, C. (2000). Prevalence of insomnia in a survey of 12,778 adults in France. Journal of Sleep Research, 9, 3542. http://dx.doi.org/10.1046/j.1365-2869.2000.00178.x

Lovibond, S. H., \& Lovibond, P. F. (1995). Manual for the depression anxiety stress scales, $\left(2^{\text {nd }}\right.$ ed.). Sydney, Australia: Psychology Foundation of Australia.

Maich, K. H. G., Lachowski, A. M., Harris, A., \& Carney, C. E. (2013, November). A preliminary psychometric exploration of the Consensus Sleep Diary. Poster presented at the meeting of the Association for Behavioural and Cognitive Therapies, Nashville, TN.

Mallon, L., Broman, J. E., \& Hetta, J. (2000). Relationship between insomnia, depression, and mortality: A 12-year follow-up of older adults in the community. International Psychogeriatrics, 12, 295-306. http://dx.doi.org/10.1017/s1041610200006414

MATLAB 8.4.0.150421. [Computer software]. (2014). Natick, MA: The MathWorks Inc.

Maxwell, J. A. (2012). A Realist Approach for Qualitative Research. Thousand Oaks, CA: Sage Publications, Inc.

Morin, C. M. (1993). Insomnia: Psychological assessment and management. New York, NY: Guilford Press.

Morin, C. M., Belleville, G., Belanger, L., \& Ivers, H. (2011). The Insomnia Severity Index: Psychometric indicators to detect insomnia cases and evaluate treatment response. Sleep, $34,601-608$.

Morin, C. M., Bootzin, R. R., Buysse, D. J., Edinger, J. D., Espie, C. A., \& Lichstein, K. L. (2006). Psychological and behavioral treatment of insomnia: Update of the recent evidence (1998-2004). Sleep, 29, 1398-1414.

Morin, C. M., LeBlanc, M., Daley, M., Gregoire, J. P., \& Merette, C. (2006). Epidemiology of 
insomnia: Prevalence, self-help treatments, consultations, and determinants of help-seeking behaviors. Sleep Medicine, 7, 123-130. http://dx.doi.org/10.1016/j.sleep.2005.08.008

Morgenthaler, T., Kramer, M., Alessi, C., Friedman, L., Boehlecke, B., Brown, T., ... \& Swick, T. (2006). Practice parameters for the psychological and behavioral treatment of insomnia: An update. An American Academy of Sleep Medicine report. Sleep, 29, 14151419. http://dx.doi.org/10.3410/f.720254864.793506780

Moss, T. G., Carney, C. E., Atwood, M.E., Crowe, B. M., \& Luminoso, K. M. (2011, November). Are people with insomnia afraid of the dark? Poster presented at the meeting of the Association for Behavioural and Cognitive Therapies, Toronto, ON.

Moss, T. G., Carney, C. E., Haynes, P., \& Harris, A. L. (2015). Is daily routine important for sleep? An Investigation of social rhythms in a clinical insomnia population. Chronobiology International, 32, 92-102. doi: 10.3109/07420528.2014.956361

Muris, P., Merckelbach, H., Mayer, B, \& Prins, E. (2000). How serious are common childhood fears? Behaviour Research and Therapy, 38, 217-228. http://dx.doi.org/10.1016/s00057967(98)00204-6

National Center for Chronic Disease and Prevention and Health Promotion, Division of Adult and Community Health. (2014). Insufficient sleep is a public health epidemic. Centers for Disease Control and Prevention. Retrieved from http://www.cdc.gov/features/dssleep/ National Sleep Foundation. (2012). 2012 bedroom poll: Summary of findings. Arlington, VA: WBA Market Research.

Nelson, D. R., Hammen, C., Daley, S. E., Burge, D., \& Davila, J. (2001). Sociotropic and autonomous personality styles: Contributions to chronic life stress. Cognitive Therapy and Research, 25, 61-76. 
Nicassio, P. M., Mendelowitz, D. R., Fussell, J. J., \& Petras, L. (1985). The phenomenology of the pre-sleep state: The development of the Pre-sleep Arousal Scale. Behavior Research and Therapy, 23, 263-271. http://dx.doi.org/10.1016/0005-7967(85)90004-x

Ohayon, M. M. (1996). Epidemiological study on insomnia in the general population. Sleep, 19(Suppl. 3), 7-15.

Ohayon, M. M. (2002). Epidemiology of insomnia: What we know and what we still need to learn. Sleep Medicine Reviews, 6, 97-111. http://dx.doi.org/10.1053/smrv.2002.0186

Ohayon, M. M., \& Shapiro, C. M. (2000). Sleep disturbances and psychiatric disorders associated with posttraumatic stress disorder in the general population. Comprehensive Psychiatry, 41, 469-478. http://dx.doi.org/10.1053/comp.2000.16568

Page, A. C., Hooke, G. R., \& Morrison, D. L. (2007). Psychometric properties of the Depression Anxiety Stress Scales (DASS) in depressed clinical samples.British Journal of Clinical Psychology, 46, 283-297. doi: 10.1348/014466500163031

Perlis, M. L., Giles, D. E., Buysse, D. J., Tu, X., \& Kupfer, D. J. (1997). Self-reported sleep disturbance as a prodromal symptom in recurrent depression. Journal of Affective Disorders, 42(2), 209-212. http://dx.doi.org/10.1016/s0165-0327(96)01411-5

Perlis, M. L., Giles, D. E., Mendelson, W. B., Bootzin, R. R., \& Wyatt, J. K. (1997). Psychophysiological insomnia: The behavioural model and a neurocognitive perspective. Journal of Sleep Research, 6, 179-188. http://dx.doi.org/10.1046/j.13652869.1997.00045.x

Pigeon, W. R., Hegel, M., Unutzer, J., Fan, M-Y., Sateia, M. J., Lyness, J. M., .. Perlis, M. L. (2008). Is insomnia a perpetuating factor for late-life depression in the IMPACT cohort? Sleep, 31, 481-488. 
Pigeon, W. R. (2010). Diagnosis, prevalence, pathways, consequences, \& treatment of insomnia. Indian Journal of Medical Research, 131, 321-332.

Pigeon, W. R., Cerulli, C., Richards, H., He, H., Perlis, M., \& Caine, E. (2011). Sleep disturbances and their association with mental health among women exposed to intimate partner violence. Journal of Women's Health, 20, 19231929. http://dx.doi.org/10.1089/jwh.2011.2781

Reiss, S. (1991). Expectancy model of fear, anxiety, and panic. Clinical Psychology Review, 11, 141-153. http://dx.doi.org/10.1016/0272-7358(91)90092-9

Roth, T. (2007). Insomnia: Definition, Prevalence, Etiology, and Consequences. Journal of Clinical Sleep Medicine, 3(Suppl. 5), 7.

Roth, T., \& Ancoli-Israel, S. (1999). Daytime consequences and correlates of insomnia in the United States: Results of the 1991 National Sleep Foundation Survey. II. Sleep, 22(Suppl. 2), 354-358.

Roth, T., Coulouvrat, C., Hajak, G., Lakoma, M. D., Sampson, N. A., Shahly, V., ... \& Kessler, R. C. (2011). Prevalence and perceived health associated with insomnia based on DSM-IVTR; International Statistical Classification of Diseases and Related Health Problems, tenth revision; and research diagnostic criteria/international classification of sleep disorders, criteria: Results from the America Insomnia Survey. Biological Psychiatry, 69, 592600. http://dx.doi.org/10.1016/j.biopsych.2010.10.023

Ross, L. E. (1961). Conditioned fear as a function of CS-UCS and probe stimulus intervals. Journal of Experimental Psychology, 61, 265273. http://dx.doi.org/10.1037/h0049071 
Rubinstein, ML, Selwyn, PA. (1998). High prevalence of insomnia in an outpatient population with HIV infection. Journal of Acquired Immune Deficiency Syndromes, 19, 260265. http://dx.doi.org/10.1097/00042560-199811010-00008

Smith, S. S., Kozak, N., \& Sullivan, K. A. (2010). An investigation of the relationship between subjective sleep quality, loneliness and mood in an Australian sample: Can daily routine explain the links?. International Journal of Social Psychiatry, 58, 166-171. doi:

\section{$10.1177 / 0020764010387551$}

Spence, K. W. \& Runquist, W. N. (1958). Temporal effects of conditioned fear on the eyelid reflex. Journal of Experimental Psychology, 55, 613-

616. http://dx.doi.org/10.1037/h0049214

Spielman, A. J., Caruso, L. S., \& Glovinsky, P. B. (1987). A behavioral perspective on insomnia treatment. Psychiatric Clinics of North America, 10, 541-553.

Stein, M. B., Belik, S. L., Jacobi, F., Sareen, J. (2008). Impairment associated with sleep problems in the community: Relationship to physical and mental health comorbidity. Psychosomatic Medicine, 70, 913-919. http://dx.doi.org/10.1097/psy.0b013e3181871405

Suka, M., Yoshida, K., \& Sugimori, H. (2003). Persistent insomnia is a predictor of hypertension in Japanese male workers. Journal of Occupational Health, 45, 344350. http://dx.doi.org/10.1539/joh.45.344

Tabachnick, B. G., \& Fidell, L. S. (2001). Using multivariate statistics (4 ${ }^{\text {th }}$ ed.). Boston, MA: Allyn and Bacon.

Taylor, D. J., Lichstein, K. L., Durrence H., Reidel, B. W., \& Bush, A. J. (2005). Epidemiology of insomnia, depression, and anxiety. Sleep, 28, 1457-1164.

Walker, D. L., Cassella, J. V., Lee, Y., De Lima, T. C. M., \& Davis, M. (1997). Opposing roles 
of the amygdala and dorsolateral periaqueductal gray in fear-potentiated startle.

Neuroscience and Biobehavioral Reviews, 21, 743-753. http://dx.doi.org/10.1016/s01497634(96)00061-9

Weathers, F. W., \& Keane, T. M. (2007). The criterion A problem revisited: Controversies and challenges in defining and measuring psychological trauma. Journal of Traumatic Stress, 20, 107-121. doi:10.1002/jts.20210

Wohlgemuth, W. K., Edinger, J. D., Fins, A. L, \& Sullivan, R. J. (1999). How many nights are enough? The short-term stability of sleep parameters in elderly insomniacs and normal sleepers. Psychophysiology, 36, 1-12. http://dx.doi.org/10.1111/1469-8986.3620233

Woodley, J., \& Smith, S. (2006). Safety behaviors and dysfunctional beliefs about sleep: Testing a cognitive model of the maintenance of insomnia. Journal of Psychosomatic Research, 60, 551-557. http://dx.doi.org/10.1016/j.jpsychores.2006.03.002

Zalai, D., Sherman, M., McShane, K., Shapiro, C. M., \& Carney, C. E. (2015). The importance of fatigue cognitions in chronic hepatitis C infection. Journal of Psychosomatic Research, 78, 193-198. http://dx.doi.org/10.1016/j.jpsychores.2014.11.011

Zayfert, C., \& DeViva, J. C. (2004). Residual insomnia following cognitive behavioral therapy for PTSD. Journal of Traumatic Stress, 17, 69-

73. http://dx.doi.org/10.1023/b:jots.0000014679.31799.e7 